\title{
Calculation of BRS Cohomology with Spectral Sequences
}

\author{
J.A. Dixon ${ }^{\star}$ \\ Theory Group, Physics Department, University of Texas, Austin, TX 78712, USA
}

Received July 11, 1989; in revised form November 15, 1990

\begin{abstract}
A method for finding the general form of the BRS cohomology space $H$ for the various gauge and supersymmetry theories is presented. The method is adapted for use in the space of integrated local polynomials of the gauge fields and ghosts with arbitrary numbers of fields and dervivatives. The technique uses the Hodge decomposition in a Fock space with a Euclidean inner product, and combines this with spectral sequences to generate simple and soluble equations whose solutions span a simple space $E_{\infty}$ isomorphic to the complicated space $H$. The technique is illustrated for pedagogic purposes by the detailed calculation of the ghost charge zero and one sectors of $H$ for Yang-Mills theory with gauge group $S O(32)$ in ten dimensions. The method is appropriate for supersymmetric theories, gravity, supergravity and superstrings where higher order terms with many derivatives occur naturally in the effective action.
\end{abstract}

\section{Table of Contents}

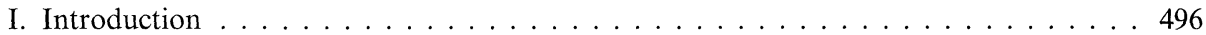

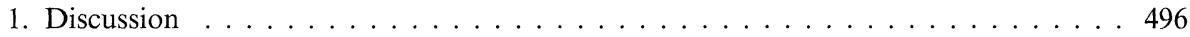

2. The BRS Identity in Yang Mills Theory . . . . . . . . . . . . . . . . . . . 499

3. Exterior Derivatives, Dimensions and the BRS Operator . . . . . . . . . . 503

II. Techniques . . . . . . . . . . . . . . . . . . . . . . . . . . . . . . . 504

4. Fock Space and Hodge Decomposition . . . . . . . . . . . . . . . . . . 504

5. Cohomology Subspaces in a Euclidean Vector Space . . . . . . . . . . . . 505

6. Spectral Sequences Defined on a Euclidean Vector Space . . . . . . . . . . . . 507

7. Using $\varepsilon$ to Incorporate Integration over Space-Time . . . . . . . . . . . . 510

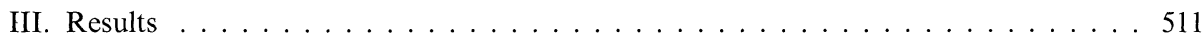

8. The Space $E_{1}$ and the Operators $\mathrm{II}_{1}, d_{1}$, and $\Delta_{1} \ldots \ldots \ldots \ldots \ldots \ldots \ldots \ldots \ldots$

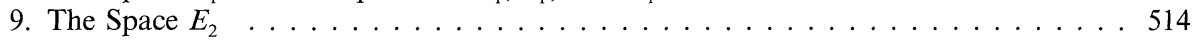

^ Research supported in part by the Robert A. Welch Foundation and NSF Grants PHY 77-18762 and PHY 9009850 


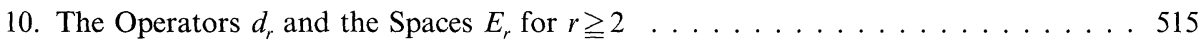

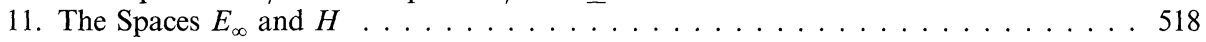

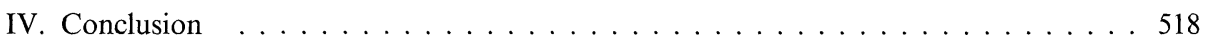

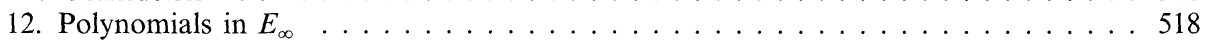

12.1. Ghost Charge Zero . . . . . . . . . . . . . . . . . . . . . . . 519

12.2. Ghost Charge One . . . . . . . . . . . . . . . . . . . 520

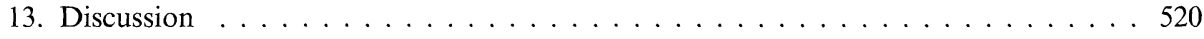

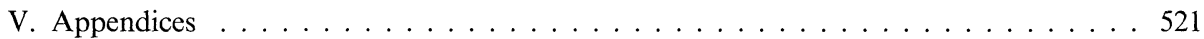

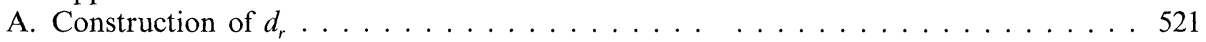

B. The Noether Procedure and the Isomorphism $E_{\infty} \rightarrow H \ldots \ldots \ldots 23$

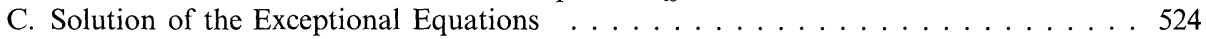

\section{Part I. Introduction}

\section{Discussion}

The purpose of this paper is to introduce and explain in a simple way a powerful general technique for calculating the local BRS cohomology of the complicated nilpotent functional differential operators that arise in local field theories [6-8, 36-39, 43]. The technique uses the Hodge decomposition in a Fock space with a Euclidean inner product, and combines this with spectral sequences to generate simple and soluble equations whose solutions span a simple space $E_{\infty}$ isomorphic to the complicated space $H$.

To fully illustrate the details of the technique and the structure of the results that are obtained with it, this article treats in detail the case of $S O$ (32) YangMills theory in 10 spacetime dimensions. This relatively complicated and very specific example is chosen partly because simpler theories in lower dimensions do not illustrate the general form of the results quite so well. Also, the 10-D $S O$ (32) Yang-Mills action is part of the supersymmetric Yang-Mills action that arises in the zero slope limit of the $N=1$ superstring theory [24]. It is hoped that the results here will be useful for the calculation of the BRS cohomology of that super Yang-Mills theory. The technique will be used in [17] for the evaluation of the local cohomology of the supersymmetric model of Wess and Zumino, where the result is curious and complicated.

The operator of interest in this paper is a simple one to write down. It is the "restricted" BRS operator:

$$
\delta=\int d^{10} x\left[D_{\mu}^{a b} \omega^{b} \frac{\delta}{\delta A_{\mu}^{a}}-\frac{1}{2} f^{a b c} \omega^{b} \omega^{c} \frac{\delta}{\delta \omega^{a}}\right],
$$

where $D_{\mu}^{a b}$ is the gauge covariant derivative (defined below) and $f^{a b c}$ are the structure constants of the Lie Algebra of the group $S O$ (32). It satisfies the usual BRS nilpotency identity:

$$
\delta^{2}=0
$$

The operator $\delta$ is assumed to act in the linear vector space of all integrated local polynomial functions of the Yang-Mills field $A$ and of the Fadeev-Popov ghost field $\omega$ and their derivatives of all orders. This space will be denoted $\mathscr{T}$. The operator is "restricted" in the sense that the actual BRS identity of the theory 
involves fermions and scalars and sources for the gauge transformations as well, as reviewed briefly below. The general unrestricted problem is closely related to the present one and an extension of the present results to that case is straightforward, although algebraic complexity becomes a problem.

The cohomology space $H$ of $\delta$ is defined to be the factor space $H$ $=\operatorname{Ker} \delta / \operatorname{Im} \delta$. A knowledge of $H$ is useful for several reasons:

(1) The objects in $H$ with ghost number $\mathscr{G}=0$ constitute the invariants of the theory. In the case of ordinary Yang-Mills theory it is easy to obtain all invariants by simple construction, but in other theories such as 10 dimensional supersymmetric Yang-Mills this question is not so easy since auxiliary fields to close the algebra and permit the development of a tensor calculus probably do not exist. I believe that the present techniques can yield useful results also in such cases, although work on this question is not yet complete. Even in ordinary Yang-Mills theory, some exceptional invariants also occur in $H$ with $\mathscr{G}=0$ : these are best thought of as generalized Chern-Simons terms and they will be described below. A complete list of the objects in $H$ with $\mathscr{G}=0$ is also essential for an understanding of the resolution of the ghost mixing problem in the renormalization of gauge invariant operators.

(2) The objects in $H$ with $\mathscr{G}=1$ constitute the anomalies that the theory may possess. My original object in solving this problem was in fact to determine whether such gauge theories as gravity, supersymmetriy and supergravity possess anomalies other than the known ones, and as far as I know this is still an open question. Obviously if heretofore undiscovered anomalies do exist it will be of interest to examine them more closely in the hope of gaining more insight into the questions of uniqueness and broken symmetry, and more generally into the nature of the theories. In fact the cohomology of the simplest supersymmetric theory is quite nontrivial [17] and it appears that there may well be previously unnoticed anomalies in supersymmetric Yang-Mills theory [18]. The techniques in this paper are used to derive the results in $[17,18]$.

(3) Objects also exist in $H$ with $\mathscr{G}=2$ and these correspond to anomalies of the kind examined in [22].

As was stated above, the existence of an object in $H$ with $\mathscr{G}=1$ constitutes a basic requirement for the existence of an anomaly in a theory. The existence of such an object does not determine whether any anomaly does in fact exist that is a matter for calculation in perturbation theory (or by other methods). However it does determine whether it is impossible for an anomaly to exist (we are considering only "Lie algebra" anomalies here - not the "global" anomalies considered for example by [42]. The test is whether the BRS cohomology space of $\mathscr{G}=1$ is empty or not. If it is empty, there can be no anomaly. If it contains nonzero objects then anomalies may exist in the theory, and they must have the same dimension, Lorentz properties and discrete symmetries as the corresponding objects in $H$. The trick is then (usually, though not always) to make their coefficients, as calculated in the theory, zero. This can sometimes be done by a judicious choice of the representations of the fermions in the theory. Another method is used in superstring theory [24], and this has been considered from a cohomological point of view by $[3,4]$.

In general one might expect that there could be anomalies of all dimensions in a given theory - not merely dimension zero in the natural units of the theory 
(see the definition of dimension in Sect. 3). Such objects would correspond to anomalies in the insertion of higher dimension invariants into the theory and would occur for example in the operator product expansion, or in the effective non-renormalizable action in a superstring theory in the zero slope limit. This phenomenon does occur in supersymmetric Yang-Mills theory [18]. It is presently not known whether the coefficients in that case are zero or not.

The present technique is designed to test for the existence of objects of this kind as well as for the usual objects with dimension zero in the natural units of the theory, which is where all known anomalies are presently located, except those in abelian theories [35].

The problem with this method of testing for the possible existence of anomalies is that finding the BRS cohomology space for a given theory is a complicated and tedious task, and it has been performed for only a very limited number of very special cases. Most papers in the literature simply restrict themselves to the dimension of the action and then consider the most general polynomials that can be written down. Obviously this does not work for theories in ten dimensions, or for supersymmetry or supergravity, since the number of terms becomes far too large. Mathematicians have been dealing with rather different but also very complicated cohomology problems for a long time. They have developed the very powerful spectral sequence method for solving this kind of problem. The purpose of the present article is to introduce the reader to this tool as adapted for the purpose of evaluating BRS cohomology spaces in gauge field theory, and to illustrate its use by calculating the cohomology space of a specific Yang-Mills theory.

Some interesting general results have been obtained concerning the cohomology of BRS operators using fibre bundle techniques in [3]. The difficulty in using the usual mathematical techniques in the solution of the field theory problem is that the usual mathematical treatments do not concern themselves with cohomology restricted to the space of local functionals of the pullback of the connection, which is essential for field theory applications. In [3], it is explained that locality is to be identified with universality as discussed therein. The connection between that result and the present method requires further examination.

Recently, a useful series of papers [10-13] has appeared. These papers give a general formula for the creation of all Lorentz invariant polynomials in the cohomology space for all compact gauge groups for the restricted BRS operator in Yang-Mills theories (and gravity).

This is a much more general result than the present paper attempts to treat, and the reader could justifiably ask whether the present more formal and complicated technique has any value. It is true that the present paper is more general in one direction - it extends the analysis to Lorentz non-invariant polynomials. But undoubtedly the analysis of Brandt et al. could be extended without a great deal of effort.

I think that the value of the present formal methods shows up best in the general analysis of the restricted BRS cohomology of a supersymmetric theory in [17]. In that case, it was not easy to guess what the form of the answer might be, but the combination of the Hodge decomposition and the spectral sequence generates a number of equations that can be (at least partially) solved to generate the answer without requiring any real insight into what is going to happen. The formality and mechanical nature of the technique is a strength in that case, whereas for the present problem it is also somewhat of a weakness. 
There is therefore some hope that these more formal methods may still be a useful tool for the terrifically difficult problem of finding the general BRS cohomology of super Yang-Mills theory, supergravity and the superstring.

A problem that is rather difficult from any known point of view is the problem of dealing with the full unrestricted BRS operator (see discussion in Sect. 2). This is a problem whose solution is needed especially in supersymmetric Yang-Mills theory in ten dimensions, for example, since there is probably no restricted operator which can be defined [16]. The following techniques are capable of dealing with the unrestricted problem, but they seem to become rather arduous and algebraically tedious, to the extent that a computer program capable of tensor manipulations appears to be a desirable addition, particularly for the unrestricted supergravity problem.

Some earlier work on the subject of this paper was included in [14 and 27]. The latter paper does not allow for the possibility of integration by parts inside integrals and consequently leaves a large part of the problem unresolved. The former papers were unpublished, and most of the material in them has been updated and included in the present work. A brief summary of the main ideas of the present paper was given in [15]. An analysis of the problem for all gauge groups in a subspace of the space $\mathscr{T}$ of all integrated polynomials has been given in [21]. Additional considerations on relating BRS symmetry to differential geometry is given in $[29,30,2,34,40]$ and many other references that I will not attempt to review here.

The spectral sequence enables one to find out the dimension of the cohomology space of given ghost charge, for a given theory, in a given spacetime dimension, for a given dimension of the relevant polynomials, and with given Lorentz and discrete properties. It does this by specifying certain rather simple polynomials in the fields with the given properties. These simple polynomials belong to a space called $E_{\infty}$. By the fundamental isomorphism theorem of spectral sequence theory, $E_{\infty} \approx H$, there must then exist corresponding non-zero polynomials in the cohomology space $H$ of the full theory.

The results obtained here for $S O$ (32) Yang-Mills theory are summarized in the conclusion, which also further discusses some of the advantages and shortcomings of the method.

\section{The BRS Identity in Yang Mills Theory}

To define the notation and make this paper reasonably self contained, we will briefly review the Ward identity for Yang-Mills theory. One starts with the gaugefixed action including ghost terms and sources for the gauge variations of the Yang-Mills field and the ghost:

$$
\begin{aligned}
S= & \int d^{D} x\left\{-\frac{1}{4} G_{\mu \nu}^{a} G^{a \mu \nu}+I[s, A]\right. \\
& \left.+\delta\left\{\xi^{a}\left[\frac{k}{2} Z^{a}-\partial^{\mu} A_{\mu}^{a}\right]-\Xi_{\mu}^{a} A^{a \mu}+K^{a} \omega^{a}\right\}\right\},
\end{aligned}
$$

which written in full using the definition of $\delta$ below becomes: 


$$
\begin{aligned}
S= & \int d^{D} x\left\{-\frac{1}{4} G_{\mu \nu}^{a} G^{a \mu \nu}+\frac{k}{2}\left(Z^{a}-\frac{1}{k} \partial \cdot A^{a}\right)^{2}\right. \\
& \left.-\frac{1}{2 k}\left(\partial \cdot A^{a}\right)^{2}+I[s, A]+\left[\Xi_{\mu}^{a}-\partial_{\mu} \xi^{a}\right] D^{\mu a b} \omega^{b}-\frac{1}{2} K^{a} f^{a b c} \omega^{b} \omega^{c}\right\} .
\end{aligned}
$$

Greek letters are used for anticommuting fields or sources and latin letters are used for commuting ones. $D$ is the dimension of space-time, $A$ is the gauge potential, $G$ is the curvature of the gauge field $A, k$ is a gauge parameter, $Z$ is an auxiliary field for the gauge fixing term, $\omega^{a}$ is the ghost, $\xi^{a}$ is the antighost and $\Xi_{\mu}^{a}$ and $K$ are sources to be used in the formulation of the Ward identity. The variations $\delta A$, etc. are defined below. The term $I[s, A]$ is meant to represent some higher dimension gauge invariant operator coupled to a source $s$ which might have Lorentz but not group indices. For example this term might be:

$$
I[s, A]=s\left[G_{\mu \nu}^{a} G^{a \mu \nu}\right]^{2} .
$$

The method to be presented deals with all possible terms $I$ in a unified way since it works to all orders in the number of derivatives on the fields and sources. For $D>4$, these terms arise naturally since the corresponding action is non-renormalizable-requiring one to add counterterms of all dimensions.

This action is invariant under the following nilpotent gauge transformations, which define the operator $\delta$ :

$$
\begin{aligned}
\delta A_{\mu}^{a}=D_{\mu}^{a b} \omega^{b}, & & \delta \omega^{a}=-\frac{1}{2} f^{a b c} \omega^{b} \omega^{c}, \\
\delta \xi^{a}=Z^{a}, & & \delta Z^{a}=0 .
\end{aligned}
$$

The sources $\Xi$ and $K$ do not transform under $\delta$. The Yang-Mills curvature is:

$$
G_{\mu \nu}^{a}=\partial_{\mu} A_{\nu}^{a}-\partial_{\nu} A_{\mu}^{a}+f^{a b c} A_{\mu}^{b} A_{\nu}^{c} .
$$

Some fifteen years ago when the BRS transformation were discovered, a number of papers were written dealing with the questions that arise from the isertion of gauge invariant local operators of high dimension [like $I(s, A)$ above] into Feynman diagrams. It was immediately evident that, in the case of non-Abelian gauge theories, such insertions generate a need for counterterms that are ghost dependent and not gauge invariant, and several papers were written dealing with the proper treatment of these counterterms [31-33, 27, 25, 26, 20, 19]. The nongauge invariant counterterms and the usual Lagrangian counterterms form part of the one-particle irreducible generating functional $\Gamma$ and can be expected in general to be the most general local polynomials in the fields and their derivatives that can be written down consistently with the dimension, conserved symmetries and BRS identities of the theory.

These BRS identities, leaving out inessential details, take (at one loop) the form $\delta \Gamma=0$, where $\delta$ is the functional dervivative operator:

$$
\begin{aligned}
\delta= & \int d^{10} x\left\{D_{\mu}^{a b} \omega^{b} \frac{\delta}{\delta A_{\mu}^{a}}-\frac{1}{2} f^{a b c} \omega^{b} \omega^{c} \frac{\delta}{\delta \omega^{a}}\right. \\
& \left.+\left[D^{v a b} G_{\mu \nu}^{b}-f^{a b c} \Xi_{\mu}^{b} \omega^{c}\right] \frac{\delta}{\delta \Xi_{\mu}^{a}}+\left[D_{\mu}^{a b} \Xi^{\mu b}+f^{a b c} K^{b} \omega^{c}\right] \frac{\delta}{\delta K^{a}}\right\} .
\end{aligned}
$$


The antighost field dependence of $\Gamma$ can be derived from the dependence of $\Gamma$ on $\Xi$ by the substitution:

$$
\Xi_{\mu}^{a} \rightarrow \Xi_{\mu}^{a}-\partial_{\mu} \xi^{a}
$$

so we have not mentioned it in the Ward identity. Note that the $\delta$ in Eq. 8 contains terms proportional to the equation of motion of the $A$ field. This $\delta$ is what we called the unrestricted operator in Sect. 1. The operator in Eq. 8 satisfies the equation $\delta^{2}=0$, reflecting the nilpotence of BRS transformations. $\Gamma$ is the generating functional of one-particle irreducible vertices for the theory, and should be considered a functional of the same fields and sources that occur in the action, which is the classical $(\hbar=0)$ approximation to $\Gamma$. The equation $\delta \Gamma=0$ imposes linear relations among the coefficients of terms in $\Gamma$ which will be satisfied in perturbation calculations provided that a regularization is used that respects the local gauge symmetry of the theory.

However, as is well known, it is often not possible to find any such regularization. This is the case in chiral Yang-Mills theory in particular. In the case where the regularization does not respect the symmetry, all that one can expect from perturbation theory is that, at one loop, the regularized $\Gamma$ will satisfy the equation

$$
\delta \Gamma=\gamma,
$$

where $\gamma$ is an integrated local polynomial functional of the fields and their derivatives (even though $\Gamma$ is of course not local). $\gamma$ will be local and its coefficients will be finite in perturbation theory as a result of the quantum action principle. This is the basic result used in [6-8] and we will ignore any problems that may relate to zero mass particles in the present, on the assumption that renormalization can be done off mass shell with no serious difficulties for present purposes. (See [5] for a relatively recent discussion of this point.) From the equations $\delta^{2}=0$ and $\delta \Gamma=0$ it follows that $\gamma$ must satisfy the equation

$$
\delta \gamma=0 \text {. }
$$

The unintegrated local polynomials in the fields, sources and their derivatives form a vector space over the real numbers. We will call this vector space $P . P$ is also the (graded) ring of polynomials in variables like:

and

$$
A_{\mu, \mu_{1} \cdots \mu_{k}}^{a}=\partial_{\mu_{1}} \cdots \partial_{\mu_{k}} A_{\mu}^{a}
$$

$$
\omega_{\mu_{1} \cdots \mu_{k}}^{a}=\partial_{\mu_{1}} \cdots \partial_{\mu_{k}} \omega^{a} \text {. }
$$

Similar considerations apply to all other fields or sources present. The integrated local polynomials $\int d^{10} x P$ also form a vector space, which we shall denote by $\mathscr{T}$. Note that $\mathscr{T}$ does not have the ring structure that $P$ does, since the product of two integrals is not an integral over the same space but over its product with an identical space. For present purposes we assume that one can always integrate by parts and discard surface terms in integrals, which is equivalent to the statement that $\mathscr{T}$ is isomorphic to the quotient space of $P$ in which two polynomials are in the same equivalence class if and only if they differ by a polynomial which is a total derivative. We can write this relationship in the form $\mathscr{T} \approx P / \partial P$. 
The operator $\delta$ acts as a linear operator on $\mathscr{T}$ or on $P$ and these spaces are both stable under the operation of $\delta$, by which we mean that $\delta \mathscr{T} \subset \mathscr{T}$ and $\delta P \subset P$.

When acting on $P, \delta$ is also an antiderivation on the ring of polynomials in the variables $A_{\mu, \mu_{1} \ldots \mu_{k}}^{a}$, etc. defined above. $\gamma$ must have ghost number $\mathscr{G}=1$ since $\delta$ increases $\mathscr{G}$ by one and $\Gamma$ itself has $\mathscr{G}=0 . \mathcal{\gamma}$ and the counterterms in $\Gamma$ are vectors in the space $\mathscr{T}$ defined above. Here we define ghost number $\mathscr{G}$ to be:

$$
\mathscr{G}=\int d^{10} x\left[\omega^{a} \frac{\delta}{\delta \omega^{a}}-\xi^{a} \frac{\delta}{\delta \xi^{a}}-\Xi_{\mu}^{a} \frac{\delta}{\delta \Xi_{\mu}^{a}}-2 K^{a} \frac{\delta}{\delta K^{a}}\right]
$$

and note the relation:

$$
[\mathscr{G}, \delta]=\delta .
$$

If there exists some $\Sigma \in \mathscr{T}$ such that $\gamma$ has the form $\gamma=\delta \Sigma$, one can always redefine the renormalization procedure in such a way that one gets the equation $\delta \Gamma^{\prime}=0$, where $\Gamma^{\prime}=\Gamma-\Sigma$. However if there exists no local $\Sigma$ which gives rise to $\gamma$ by a relation of this form it is not possible to maintain the BRS identity upon renormalization. Then the theory is said to possess an anomaly. No matter how one regularizes and renormalizes one will always get $\delta \Gamma=\gamma$ for some nonzero $\gamma$. The precise form of $\gamma$ can be changed by a redefinition of the renormalization of $\Gamma$, but $\gamma$ cannot be entirely eliminated.

The cohomology space $H_{P}\left(H_{\mathscr{T}}\right)$ of $\delta$ in the vector space $P(\mathscr{T})$ is defined to be the factor space of solutions of $\delta P=0(\Delta \mathscr{T}=0)$ modulo polynomials of the form $\delta P(\delta \mathscr{T})$. From the above discussion we see that anomalies in the theory correspond to elements of $H_{\mathscr{T}}$ with ghost charge one. Evaluation of $H_{\mathscr{T}}$ turns out to be much more difficult than the evaluation of $H_{P}$.

If the relevant part of the cohomology space with $\mathscr{G}=1$ is empty or if the coefficient of the cohomology class calculated in perturbation theory is zero, one can redefine $\Gamma$ so that $\delta \Gamma=0$. Then one must confront the issues arising from the presence of gauge non-invarant counterterms in $\Gamma$. The first problem that arises is the fact that typically a large number of non-gauge invariant and ghost dependent counterterms are needed for the renormalization of operators like $I$ above in Eq. 5. This raises a concern that the anomalous dimensions of higher dimension gauge invariant operators might be gauge dependent since the gauge invariant operators mix upon renormalization with gauge non-invariant operators.

The natural conjecture to make in order to allay this concern is that the ghost charge zero sector of $H_{\mathscr{T}}$ is spanned by the integrals of local gauge-invariant ghost-independent polynomials. If this is true then one can expect that the anomalous dimensions of gauge invariant operators will be gauge invariant. If it is not true then there could be serious ghost-mixing problems in the theory which might cause problems with unitarity. This is an issue whose resolution in the case of theories like supergravity could conceivably have interesting problems.

Gravity and supergravity give rise to very similar problems and questions. In these cases it is not necessary to consider the insertion of large dimension gauge invariant operators to generate the general problem however. The dimensional coupling constant in these non-renormalizable theories make the consideration of polynomials of all dimensions inevitable. This is of course also the case for Yang-Mills theory in ten space-time dimensions. 
Examination of any specific gauge theory reveals that $\delta$ is a complicated operator and that generally the analysis of its local cohomology is a forbidding task. When performed even with a limitation of operators to those with dimension less than five, it is algebraically very tedious. It has sometimes been suggested that use of the "background field" method can avoid the problems of non-gaugeinvariant conterterms, but this is not true since these counterterms still occur in background field calculations whenever vertices containing only quantized fields appear.

The spectral sequence technique for finding the cohomology space resembles perturbation theory since it can be construed as a set of successive approximations in powers of the coupling constant. However in the present case, unlike in perturbation theory, the approximation converges after a few steps to an exact answer.

\section{Exterior Derivatives, Dimensions and the BRS Operator}

The rest of this paper is devoted to the analysis of the cohomology of the restricted operator $\delta$ in Eq. (1) in the space $\mathscr{T}$ of integrated local polynomials in $A, \omega$ and their derivatives.

It is easy to see that $\delta$ satisfies the relation:

$$
\left[\delta, \partial_{\mu}\right]=0
$$

where the operator $\partial_{\mu}$ is to be understood as the operator that can be represented by:

$$
\partial_{\mu}=\int d^{D} x\left\{\partial_{\mu} \omega^{a} \frac{\delta}{\delta \omega^{a}}+\partial_{\mu} A_{v}^{a} \frac{\delta}{\delta A_{v}^{a}}\right\} .
$$

It follows from the above that if we define the operator $\delta_{\varepsilon}$ by:

$$
\delta_{\varepsilon}=\delta+\varepsilon^{\mu} \partial_{\mu},
$$

where $\varepsilon_{\mu}$ is a constant (i.e. spacetime independent) anticommuting Lorentz vector, then:

$$
\delta_{\varepsilon}^{2}=0
$$

Note that the term $\partial_{\mu}$ gives zero on any integrated functional, since it is just the derivative operator on the functions being integrated. In Sect. 7, we show that the cohomology of $\delta_{\varepsilon}$ acting on the space $P_{\varepsilon}$ (the space $P$ augmented with a dependence on $\varepsilon_{\mu}$ ), is isomorphic to the cohomology of $\delta$ acting on the space $\mathscr{T}$. Moreover, we will use this in our calculations because the cohomology of $\delta_{\varepsilon}$ is more amenable to calculation using the present methods. Let us assign the following dimensions $\mathscr{D}$ to the fields: $\mathscr{D}(A)=1, \mathscr{D}(\omega)=0, \mathscr{D}(\varepsilon)=-1$, $\mathscr{D}(\partial)=1, \mathscr{D}(x)=-1$. Let us assume that all coupling constants are absorbed into the definitions of the fields. Then the action (Eq. 3) has $\mathscr{D}(S)=4-D$, and the operator $\delta_{\varepsilon}$ has $\mathscr{D}=0$ which can be expressed in the form:

$$
\left[\mathscr{D}, \delta_{\varepsilon}\right]=0 \text {. }
$$


Now we can assert:

Theorem 1. The space of all polynomials of a given dimension in the space $P_{\varepsilon}$ (defined to be the space $P$ augmented with a dependence on the $\varepsilon$ variable) is $a$ finite dimensional vector space and $\delta_{\varepsilon}$ has no matrix elements between subspaces of different dimension.

Proof. $P_{\varepsilon}$ can contain a maximum of $D$ powers of $\varepsilon$ and a maximum of $16 \times 31$ powers of underived $\omega$ (for the present example), since both of these are anticommuting parameters, the spacetime has $D$ dimensions and the group $S O$ (32) has $16 \times 31$ parameters. The remaining variables are $A_{\mu}^{a}$ and $\partial_{\mu}$ both of which have positive dimension, so that only a finite number of combinations with a given dimension are available using these variables. Finally, since $\delta_{\varepsilon}$ commutes with the dimension, it clearly has zero matrix elements between subspaces of different dimensions. QED

A similar theorem and proof hold for the space $\mathscr{T}$. The use of this theorem lies in the fact that it means that all our problems are effectively finite-dimensional problems, with no difficult issues arising from infinite dimensional vector spaces.

\section{Part II. Techniques}

\section{Fock Space and Hodge Decomposition}

As already mentioned we will work in the space $P$ of unintegrated polynomials in $\partial$ and the fields $A$ and $\omega$ and their derivatives. The operator $\partial_{\mu}$ mentioned in Sect. 3 is zero when acting in $\mathscr{T}$ but it is not zero when acting in $P$. A typical independent tensor variable in the space $P$ is $A_{\mu, \mu_{1} \ldots \mu_{k}}^{a}$ defined by Eq. 12. This expression is symmetric under all permutations of $1,2 \cdots k$ because derivatives commute. Note that this tensor has no symmetriy between its first spacetime index and the rest. This fact plays a crucial role. More generally, the present technique amounts to little more than a systematic way of keeping track of this kind of symmetry, and all the difficulties that arise in more complicated $\delta$ 's for supergravity, etc. only arise from more complicated symmetries of this type, plus some extra difficulties arising from making these kinds of tensors traceless when equation of motion terms are present in the unrestricted operators. $\delta$ can be written in terms of the variables defined above (and analogous ones for all the other fields and sources) by defining an adjoint "destruction operator" to satisfy:

$$
\begin{aligned}
& {\left[\left(A_{\nu, \nu_{1} \cdots v_{k}}^{b}\right)^{\dagger}, A_{\mu, \mu_{1} \cdots \mu_{k}}^{a}\right]} \\
& \quad=\delta_{b}^{a} \delta_{\mu}^{v} \sum_{\text {permutations }}\left\{\delta_{\mu_{1}}^{\nu_{1}} \delta_{\mu_{2}}^{\nu_{2}} \cdots \delta_{\mu_{k}}^{v_{k}}+\delta_{\mu_{1}}^{\nu_{2}} \delta_{\mu_{2}}^{\nu_{1}} \cdots \delta_{\mu_{k}}^{\nu_{k}}+\text { etc. }\right\} \\
& \left\{\left(\omega_{\nu_{1} \cdots v_{k}}^{b}\right)^{\dagger}, \omega_{\mu_{1} \cdots \mu_{k}}^{a}\right\} \\
& \quad=\delta_{b}^{a} \sum_{\text {permutations }}\left\{\delta_{\mu_{1}}^{\nu_{1}} \delta_{\mu_{2}}^{\nu_{2}} \cdots \delta_{\mu_{k}}^{v_{k}}+\delta_{\mu_{1}}^{\nu_{2}} \delta_{\mu_{2}}^{\nu_{1}} \cdots \delta_{\mu_{k}}^{\nu_{k}}+\text { etc. }\right\}
\end{aligned}
$$

where the notation $\delta_{\mu}^{v}$ indicates a tensor which is one when $\mu=v$ and is zero otherwise, and where the $k$ ! permutations make the sum totally symmetric in 1 , $2 \cdots k$. Let us now consider the first part of the operator in Eq. (1) whose 
cohomology we want to compute. In the space $P$ we have the equivalence:

$$
\delta_{0}=\int d^{D} x \partial_{\mu} \omega^{a} \frac{\delta}{\delta A_{\mu}^{a}}=\sum_{k=1}^{\infty} \frac{1}{(k-1) !} \omega_{\mu_{1} \mu_{2} \cdots \mu_{k}}^{a}\left(A_{\mu_{1}, \mu_{2} \cdots \mu_{k}}^{a}\right)^{\dagger} .
$$

The space of polynomials in these variables and their adjoints is a Fock space of the kind familiar in quantum field theory. It has a positive definite inner product and we can write its vectors as kets (bras) which result from creation (destruction) operators acting on the vacuum state. For example we have:

$$
\left|A_{\mu}^{a} \omega^{b}\right\rangle=A_{\mu}^{a} \omega^{b}|0\rangle
$$

and the inner product of a bra and a ket is given for example by:

$$
\left\langle A_{\mu}^{a} \omega^{b} \mid A_{\nu}^{c} \omega^{d}\right\rangle=\left\langle 0\left|\left(A_{\mu}^{a} \omega^{b}\right)^{\dagger} A_{\nu}^{c} \omega^{d}\right| 0\right\rangle=\delta_{a}^{c} \delta_{b}^{d} \delta_{\nu}^{\mu} .
$$

Note that the destruction operators are the ones that have a $\uparrow$ contrary to the usual convention. The adjoint operator $\left(\delta_{0}\right)^{\dagger}$ is given by:

$$
\left(\delta_{0}\right)^{\dagger}=\sum_{k=1}^{\infty} \frac{1}{(k-1) !}\left(A_{\mu_{1}, \mu_{2} \cdots \mu_{k}}^{a}\right)\left(\omega_{\mu_{1} \mu_{2} \cdots \mu_{k}}^{a}\right)^{\dagger} .
$$

A concern that might arise is whether one is constrained to maintain Lorentz invariance by using the indefinite Lorentz metric instead of Kronecker deltas in these definitions. In fact no such problem arises since we can define any inner product we want - we are not trying to compute $S$-matrix elements here. We are solving a purely algebraic problem.

\section{Cohomology Subspaces in a Euclidean Vector Space}

The cohomology space $H$ of a nilpotent operator $\delta$ in a finite dimensional inner product space can be characterized in a simple way. Essentially one uses the Hodge decomposition, which notes the following isomorphisms which are easily demonstrated for finite dimensional vector subspaces:

$$
\begin{aligned}
H= & \operatorname{Ker} \delta / \operatorname{Im} \delta \approx(\operatorname{Ker} \delta) \cap(\operatorname{Im} \delta)^{\perp} \approx(\operatorname{Ker} \delta) \cap\left(\operatorname{Ker} \delta^{\dagger}\right) \\
& \approx \operatorname{Ker}\left(\delta+\delta^{\dagger}\right) \approx \operatorname{Ker}\left(\delta+\delta^{\dagger}\right)^{2} \approx \operatorname{Ker} \Delta=E
\end{aligned}
$$

where we define the "Laplacian" operator $\Delta$ for a given $\delta$ by:

$$
\Delta=\left(\delta+\delta^{\dagger}\right)^{2}=\delta \delta^{\dagger}+\delta^{\dagger} \delta
$$

and we define $E$ as its kernel. For example the identity $(\operatorname{Im} \delta)^{\perp}=\operatorname{Ker} \delta^{\dagger}$ can be proved as follows (the notation $A \Leftrightarrow B$ means $A$ implies $B$ and vice versa):

$$
\begin{aligned}
& x \in \operatorname{Ker} \delta^{\dagger} \Leftrightarrow \delta^{\dagger} x=0 \Leftrightarrow\left\langle y \mid \delta^{\dagger} x\right\rangle=0 \text { for all } y \\
& \Leftrightarrow\langle\delta y \mid x\rangle=0 \text { for all } y \Leftrightarrow x \in(\operatorname{Im} \delta)^{\perp} .
\end{aligned}
$$

The operator $\Delta$ has some very useful properties. It is hermitian and positive semidefinite, and frequently it can be written as the sum of hermitian positive semidefinite terms, which therefore must individually be zero if $\Delta$ is zero, thus yielding 
a large set of equations which turn out to be easily solved when things work out nicely. Things do work out very nicely in the present case. In general it can be difficult to see exactly how to express $\Delta$ as a sum of useful positive semi-definite forms - but with some work it usually becomes clear. In the case of $\delta_{0}$ defined in Eq. (23) and its adjoint Eq. (26), computation reveals that:

where

$$
\begin{aligned}
\Delta_{0}= & \sum_{k=1}^{\infty} \frac{1}{k !}\left\{\omega_{\mu_{1} \mu_{2} \cdots \mu_{k}}^{a}\left(\omega_{\mu_{1} \mu_{2} \cdots \mu_{k}}^{a}\right)^{\dagger}\right. \\
& \left.+k \tilde{A}_{\mu_{1}, \mu_{2} \cdots \mu_{k}}^{a}\left(\tilde{A}_{\mu_{1}, \mu_{2} \cdots \mu_{k}}^{a}\right)^{\dagger}\right\}
\end{aligned}
$$

$$
\tilde{A}_{\mu_{1}, \mu_{2} \cdots \mu_{k}}^{a}=\frac{1}{k} \sum_{i=1}^{k} A_{\mu_{t}, \mu_{1} \mu_{2} \cdots \hat{\mu}_{t} \cdots \mu_{k}}^{a} .
$$

Note that $\Delta_{0}$ is the sum of terms of the form $Q_{i}\left(Q_{l}\right)^{\dagger}$ so that $E_{1}=\operatorname{Ker} \Delta_{0}$ must satisfy the equations $Q_{i}^{\dagger} E_{1}=0$ for each $i$. Specifically

$$
\begin{aligned}
& \left(\omega_{\mu_{1} \mu_{2} \cdots \mu_{k}}^{a}\right)^{\dagger} E_{1}=0, \\
& \left(\tilde{A}_{\mu_{1}, \mu_{2} \cdots \mu_{k}}^{a}\right)^{\dagger} E_{1}=0
\end{aligned}
$$

for all $k=1,2, \ldots$ It follows that $E_{1}$ is spanned by the set of all monomials consisting of products of $\omega^{a}$ (with no spacetime indices) and of:

$$
A_{\mu_{1}, \mu_{2} \cdots \mu_{k}}^{\prime a}=A_{\mu_{1}, \mu_{2} \cdots \mu_{k}}^{a}-\tilde{A}_{\mu_{1}, \mu_{2} \cdots \mu_{k}}^{a} .
$$

Now recall the isomorphism $H_{0} \approx E_{1}$, which follows from Eq. (27) and the definitions:

$$
\begin{gathered}
H_{0}=\operatorname{Ker} \delta_{0} / \operatorname{Im} \delta_{0} \\
E_{1}=\operatorname{Ker} \Delta_{0}=\operatorname{Ker}\left(\delta_{0}+\delta_{0}^{\dagger}\right)^{2}
\end{gathered}
$$

It has been shown that the space $H_{0}$ is isomorphic to the subspace $E_{1}$ generated by all polynomials which depend only on underived $\omega$ (i.e. no spacetime indices) and on the fields $A^{\prime}$ defined above.

Two problems must be solved to deal with the problem of interest:

1. Integration over $x$ must be included in the problem so that we can work in $\mathscr{T}$ rather than in $P$.

2. When the nonlinear terms in $\delta$ are included, $\Delta$ computed from $\delta$ is not simple and it is not possible to characterize its kernel in a straightforward way.

To deal with problem 1 in our context we use the result proved in Sect. 7. Problem 2 is solved with spectral sequences, which are introduced in Sect. 6. They are also used to demonstrate the result in Sect. 7. 


\section{Spectral Sequences Defined on a Euclidean Vector Space}

Note that the operator $\delta_{\varepsilon}$ defined by

$$
\delta_{\varepsilon}=\int d^{10} x\left[D_{\mu}^{a b} \omega^{b} \frac{\delta}{\delta A_{\mu}^{a}}-\frac{1}{2} f^{a b c} \omega^{b} \omega^{c} \frac{\delta}{\delta \omega^{a}}\right]+\varepsilon^{\mu} \partial_{\mu}
$$

can be written in the form $\delta_{\varepsilon}=\delta_{0}+\delta_{1}$, where

with

$$
\left[N, \delta_{i}\right]=i \delta_{i}
$$

$$
N=N(A)+N(\omega)+N(\varepsilon) .
$$

Here we define the $N(f)$ operators to count the number of fields of the indicated kind:

$$
N(f)=\int d^{D} x f \frac{\delta}{\delta f},
$$

except of course that $N(\varepsilon)=\varepsilon_{\mu}\left(\varepsilon_{\mu}\right)^{\dagger}$ has no integration. Specifically we have:

$$
\begin{gathered}
\delta_{0}=\int d^{10} x\left[\partial_{\mu} \omega^{a} \frac{\delta}{\delta A_{\mu}^{a}},\right. \\
\delta_{1}=\int d^{10} x\left[f^{a b c} A_{\mu}^{b} \omega^{c} \frac{\delta}{\delta A_{\mu}^{a}}-\frac{1}{2} f^{a b c} \omega^{b} \omega^{c} \frac{\delta}{\delta \omega^{a}}\right]+\varepsilon^{\mu} \partial_{\mu} .
\end{gathered}
$$

We can decompose any polynomial in the form (direct sum):

where

$$
P=\sum_{i=0}^{\infty} P_{i}
$$

$$
N P_{l}=i P_{i} .
$$

$N$ is said to provide a grading of the space $P$. It just counts the number of fields plus the number of powers of $\varepsilon$. The cohomology of $\delta_{0}$ was analyzed above.

Once a grading is chosen with properties like those above one can compute the spectral sequence corresponding to that grading. The spectral sequence consists of a sequence $(r=0,1,2 \cdots)$ of nilpotent operators denoted $d_{r}$ and their corresponding cohomology subspaces denoted $E_{r+1}$. The original space $P$ is the first space $E_{0}$. The operators $d_{r}$ and the spaces $E_{r}$ are determined by the grading $N$ and the original operator $\delta$. Each successive cohomology space $E_{r+1}$ is a subspace of the previous one $E_{r}$. Each successive nilpotent operator $d_{r}$ acts stably in the subspace $E_{r}$. In all the cases of interest for us at present it turns out that $d$, is identically equal to zero for all $r \geqq q$ for some finite positive integer $q$. In the case examined in this paper, $q=6$. As a result all the spaces $E_{r}$ for $r \geqq q$ are identical to $E_{q}$. This final space $E_{q}$ is also called $E_{\infty}$ since it is the limit of the sequence. The sequence is said to collapse to $E_{\infty}$.

This ultimate cohomology space $E_{\infty}$ can be proved to be isomorphic to the cohomology space $H$ of the original operator $\delta$. The isomorphism preserves properties like dimension, Lorentz character, discrete symmetries, ghost number, 
etc., so long as these are preserved by $N$. Hence it is easy to describe the main features of the original cohomology space in terms of the simple one. Now we give the form of these spaces explicitly.

First we define filtered subspaces $F_{i}$ and $F^{l}$ of $P$ by:

$$
\begin{aligned}
& F_{l}=\sum_{j \geqq i} P_{J}=P_{i}+P_{\imath+1}+P_{i+2}+\cdots, \\
& F^{i}=\sum_{j \leqq i} P_{J}=P_{i}+P_{\imath-1}+P_{i-2}+\cdots .
\end{aligned}
$$

It is easy to verify that $F_{l}$ is stable under $\delta$ :

$$
\delta F_{\imath} \subset F_{i},
$$

and that $F^{i}$ is stable under $\delta^{\dagger}$. Normally spectral sequences are defined as factor spaces [23] but in the present case where the space has an inner product we can use the standard isomorphism between a factor space and the corresponding subspace ( $M$ and $N$ stand for any subspaces such that $N \subset M$ ):

$$
M / N \approx M \cap N^{\perp}
$$

to vary the usual definition to the following:

$$
\begin{gathered}
E_{r}^{p}=P_{p} \cap\left(\delta F_{p-r+1} \cap F_{p}\right)^{\perp} \cap\left(\delta^{\dagger} F^{p+r-1} \cap F^{p}\right)^{\perp} \\
E_{r}=\sum_{p} E_{r}^{p}
\end{gathered}
$$

The spaces $E_{r}$ are the subspaces of the spectral sequence. However it takes some effort to extract information from the definition. It can be shown using standard arguments for spectral sequences [23] that $\delta$ induces a series of coboundary operators $d_{r}$ acting on $E_{r}$ satisfying the relations:

$$
\begin{gathered}
{\left[N, d_{r}\right]=r d_{r},} \\
{\left[\mathscr{F}, d_{r}\right]=d_{r},} \\
{\left[d_{r}\right]^{2}=0,}
\end{gathered}
$$

where $\mathscr{F}$ is defined to be the generalization of the ghost charge $\mathscr{G}$ appropriate to the operator $\delta_{\varepsilon}$ rather than $\delta$ :

$$
\begin{gathered}
\mathscr{G}=N(\omega), \\
\mathscr{F}=N(\omega)+N(\varepsilon),
\end{gathered}
$$

and it satisfies the relation:

$$
\left[\mathscr{F}, \delta_{\varepsilon}\right]=\delta_{\varepsilon}
$$

The most important feature of the sequence of subspaces $E_{r}$ is that each one is the cohomology space of the previous one:

$$
E_{r+1}=\operatorname{Ker} \Delta_{r} \cap E_{r},
$$


where

$$
\Delta_{r}=\left(d_{r}+d_{r}^{\dagger}\right)^{2}
$$

Moreover the sequence of subspaces $E_{r}$ converges to a subspace $E_{\infty}$ after a finite number (which we will call $q$ ) of steps, and the cohomology space of $\delta$ is isomorphic to $E_{q}$ :

$$
H \approx \operatorname{Ker} \Delta \approx E_{\infty}=E_{q} .
$$

The operators $d_{r}$ can be written explicitly in terms of the Fock space expressions for $\delta_{0}$ and $\delta_{1}$ as follows:

$$
\begin{gathered}
d_{0}=\delta_{0}, \\
d_{1}=\Pi_{1} \delta_{1} \Pi_{1}, \\
d_{2}=\Pi_{2}\left\{\delta_{1} \frac{\delta_{0}^{\dagger}}{\Delta_{0}} \delta_{1}-\delta_{2}\right\} \Pi_{2}, \\
d_{3}=\Pi_{3}\left\{\delta_{1} \frac{\delta_{0}^{\dagger}}{\Delta_{0}} \delta_{1} \frac{\delta_{1}^{\dagger}}{\Delta_{1}} \delta_{1} \frac{\delta_{0}^{\dagger}}{\Delta_{0}} \delta_{1}-\delta_{1} \frac{\delta_{0}^{\dagger}}{\Delta_{0}} \delta_{1} \frac{\delta_{0}^{\dagger}}{\Delta_{0}} \delta_{1}\right. \\
\left.-\delta_{2} \frac{\delta_{0}^{\dagger}}{\Delta_{0}} \delta_{1}-\delta_{1} \frac{\delta_{0}^{\dagger}}{\Delta_{0}} \delta_{2}+\delta_{3}\right\} \Pi_{3} .
\end{gathered}
$$

In the above formulae we have envisaged the possibility that $\delta$ has more than two terms in Eq. (38), since this will be useful in future work. These formulae evidently become increasingly complicated as $r$ increases.

In the above $\Pi_{r}$ are orthogonal projection operators onto the spaces $E_{r}$, which means that they satisfy the relations:

$$
\begin{gathered}
\Pi_{r}^{2}=\Pi_{r}=\Pi_{r}^{\dagger}, \\
\Pi_{r} E_{r}=E_{r},
\end{gathered}
$$

and $\frac{1}{\Delta_{r}}$ are generalized inverses which satisfy the equations:

$$
\frac{1}{\Delta_{r}} \Delta_{r}=\Pi_{r}-\Pi_{r+1} .
$$

Fortunately and contrary to appearances from the above, $d_{r}$ becomes very simple in practice as $r$ gets larger, and indeed vanishes indentically for all $r$ greater than or equal to some value $q$ which depends on the Yang-Mills group and the dimension of spacetime. In fact in Yang-Mills and gravity, it is clear that most of the hard work is done once one has analyzed $E_{1}$ and $E_{2}$.

These formulae can be derived by analyzing the definition of $E_{r}^{p}$ given above. See Appendix A for details. 


\section{Using $\varepsilon$ to Incorporate Integration over Space-time}

We want to calculate the cohomology of $\delta$ on the space $\mathscr{T}$ of integrated polynomials. Note that the equation:

$$
\delta \int d^{D} x P=0
$$

implies that $\delta P=\partial_{\mu} P^{\mu}$ for some $P^{\mu}$ contained in the space $P$.

Now that we have introduced the Fock space and the spectral sequence, we can prove the following theorem, also discussed in $[2,21]$, which is essential for further progress:

Theorem 2. The cohomology space of the operator $\delta$ acting in the space $I$ of integrated local polynomials is isomorphic to the cohomology space of $\boldsymbol{\delta}_{\varepsilon}$ acting in the space $P_{\varepsilon}$ of unintegrated local polynomials augmented by a dependence on the variable $\varepsilon$. Here $\mathscr{T}$ is defined by the property that the integral of a total derivative $\int d D x \partial_{\mu} P^{\mu}$ is taken to be zero, so that $\mathscr{T} \approx P / \partial_{\mu} P^{\mu}$.

Proof. Consider the spectral sequence for $\delta_{\varepsilon}$ generated by the grading operator $N=\mathscr{G}$, where $\mathscr{G}$ is the ghost number in Eq. (54). Then

where

$$
\delta_{\varepsilon}=\delta_{0}+\delta_{1}
$$

and

$$
\delta_{0}=\varepsilon \cdot \partial
$$

$$
\delta_{1}=\delta
$$

We now compute the Laplacian:

$$
\Delta_{0}=\left(\delta_{0}+\delta_{0}^{\dagger}\right)^{2}=\varepsilon^{\mu \dagger} \varepsilon^{\nu}\left[\partial_{\mu}^{\dagger}, \partial_{\nu}\right]+\partial_{\mu}\left(\partial_{\mu}\right)^{\dagger}=\varepsilon_{\mu}^{\dagger} \varepsilon_{\mu} \tilde{N}+\partial_{\mu}\left(\partial_{\mu}\right)^{\dagger},
$$

where the result is obtained by writing out the operators in Fock space form and doing a straightforward computation. The operator $\tilde{N}$ is defined by:

$$
\tilde{N}=N(A)+\mathrm{N}(\omega) \text {. }
$$

The computation uses the following identity:

$$
\left[\partial_{\mu}^{\dagger}, \partial_{\nu}\right]=\delta_{\nu}^{\mu} \tilde{N}
$$

which is easily derived from the following representation for the operator $\partial$ :

$$
\partial_{\mu}=\sum_{k=0}^{\infty} \frac{1}{k !}\left[A_{v, \mu_{1} \mu_{2} \cdots \mu_{k} \mu}^{a}\left(A_{v, \mu_{1} \mu_{2} \cdots \mu_{k}}^{a}\right)^{\dagger}+\omega_{\mu_{1} \mu_{2} \cdots \mu_{k} \mu}^{a}\left(\omega_{\mu_{1} \mu_{2} \cdots \mu_{k}}^{a}\right)^{\dagger}\right]
$$

Now consider the space $E_{1}=\operatorname{Ker} \Delta_{0}$, where $\Delta_{0}$ is given in Eq. (71). It consists of local polynomial functions $P=P(A, \omega, \partial)$ of the fields $A$ and $\omega$ multiplied (in ten spacetime dimensions) by the term $\varepsilon^{10}$ defined by:

$$
\varepsilon^{10}=\varepsilon_{0} \varepsilon_{1} \cdots \varepsilon_{9}
$$

and satisfying the identity:

$$
\left(\partial_{\mu}\right)^{\dagger} P=0
$$


In other words we have the following form for $E_{1}$ :

$$
E_{1}=P(A, \omega) \varepsilon^{10},
$$

where $P$ is subject to $\left(\partial_{\mu}\right)^{\dagger} P=0$. It is actually possible to find a simple form for the projection operator $\Pi_{1}$ for this case, but it does not appear to be very useful in practice, so we will not discuss it here. Consider now the operator $d_{1}$ defined by Eq. (61). Note that the cohomology of $d_{1}$ is the cohomology of the operator $\delta$ restricted to the subspace of local polynomials which are orthogonal to the local polynomials that can be expressed in the form:

$$
P=\partial_{\mu} P^{\mu}
$$

for some other local polynomial $P^{\mu}$. It is clear that $d_{r}$ is zero in this case for $r \geqq 2$, since the equations:

imply that

$$
\begin{gathered}
{\left[N, d_{r}\right]=\left[\mathscr{G}, d_{r}\right]=r d_{r},} \\
{\left[\mathscr{F}, d_{r}\right]=d_{r}}
\end{gathered}
$$

$$
\left[N(\varepsilon), d_{r}\right]=(1-r) d_{r}
$$

which means that $d_{r}$ changes the eigenvalue of $N(\varepsilon)$ by $1-r$, but since all of $E_{1}$ (and so $E_{r}$ for $r \geqq 1$, since $E_{r+1} \subset E_{r}$ ) is restricted to the subspace with $N(\varepsilon)=D$, this can only hold if $r=1$ or if $d_{r}=0$, which establishes the result.

It follows that the cohomology of $\delta_{\varepsilon}$ on the space $P_{\varepsilon}$ is isomorphic to the cohomology of $d_{1}$ on the space $E_{1}$. Now note that there is an isomorphism $\Pi_{1}^{\prime}$ [from Eq. (48)] which maps $\mathscr{T}$ into $E_{1}$ :

$$
\Pi_{1}^{\prime}: \frac{P}{\partial_{\mu} P^{\mu}} \approx \mathscr{T} \rightarrow E_{1}=\left[\partial_{\mu} P^{\mu}\right]^{\perp}
$$

Next consider the operator $\delta_{\mathscr{T}}$ induced by $\delta$ under the projection $P_{\mathscr{T}}: P \rightarrow \mathscr{T}=P /$ $\partial P$. It is uniquely defined because the subspace $\partial P$ is stable under $\delta$ [from Eq. (16)]. The following identities evidently hold:

$$
\begin{gathered}
\Pi_{1}^{\prime} P_{\mathscr{T}}=\Pi_{1}: P \rightarrow E_{1}, \\
\Pi_{1}^{\prime} \delta_{\mathscr{T}}=d_{1} \Pi_{1}^{\prime}: \mathscr{T} \rightarrow E_{1} .
\end{gathered}
$$

Therefore the isomorphism $\mathscr{T} \rightarrow \mathrm{E}_{1}$ is an isomorphism of differential spaces and consequently the homology spaces are also isomorphic [23]:

$$
H_{\mathscr{T}}(\delta) \approx E_{2} \approx H_{P_{\varepsilon}}\left(\delta_{\varepsilon}\right)
$$

which is what the theorem states. QED

\section{Part III. Results}

Now we can proceed to calculate the cohomology space for the restricted BRS operator for $S O(32)$ Yang-Mills theory in ten dimensions. 
8. The Space $E_{1}$ and the Operators $\Pi_{1}, d_{1}$, and $\Delta_{1}$

At first glance, it would appear that a good spectral sequence would be that used in Sect. 7, but in fact that one turns out to be hard to use in practice. The most useful spectral sequence for the present case appears to be that generated by the grading operator $N$ in Eq. (39). With this choice $N, \delta$ splits up as indicated in Eq. 41 and 42 .

The computation of $\Delta_{0}$ is easy and was completed above. One deduces Eq. (32) and (33).

When we evaluate $d_{1}$, we will need to know some more detailed properties of the operator $\Pi_{1}$ which projects onto the subspace $E_{1}=\operatorname{Ker} \Delta_{0}$. This operator can be constructed explicitly as an infinite series in the normal ordered Fock space operators. The operator $\Pi_{1}$ takes the form:

$$
\Pi_{1}=\sum_{k=0}^{\infty} N_{k}
$$

where $N_{k}$ is defined by:

$$
N_{k+1}=N_{1} N_{k}-k N_{k}
$$

and this iterative definition is started with:

$$
\begin{gathered}
N_{0}=1, \\
N_{1}=\sum_{i=1}^{\infty} \frac{1}{(i-1) !} \tilde{A}_{\mu_{1} \mu_{2} \ldots \mu_{i}}^{a}\left(\tilde{A}_{\mu_{1} \mu_{2} \cdots \mu_{2}}^{a}\right)^{\dagger} .
\end{gathered}
$$

This operator $\Pi_{1}$ satisfies the relations 64 . Another way of looking at the space $E_{1}$ is by considering the basis of variables out of which it is constructed. The space $E_{1}$ consists of all polynomials constructed from the physical fields $A^{\prime}$, the underived ghost field $\omega$ and the anticommuting vector $\varepsilon$. We can write this succinctly in the form:

$$
E_{1}=E_{1}\left(\omega^{a}, A_{\mu, \mu_{1} \mu_{2} \cdots \mu_{k}}^{\prime a}, \varepsilon\right)
$$

Now we proceed to the operator $d_{1}$ and the space $E_{2}$. It follows from Eq. (42) and (61) that the operator $d_{1}$ takes the form:

$$
d_{1}=\Pi_{1}\left\{\int d^{10} x\left[f^{a b c} A_{\mu}^{b} \omega^{c} \frac{\delta}{\delta A_{\mu}^{a}}-\frac{1}{2} f^{a b c} \omega^{b} \omega^{c} \frac{\delta}{\delta \omega^{a}}\right]+\varepsilon^{\mu} \partial_{\mu}\right\} \Pi_{1} .
$$

This formula for $d_{1}$ can be written more succinctly as:

$$
d_{1}=\Pi_{1}\left\{\omega^{a}\left[T^{a}+\frac{1}{2} Y^{a}\right]+\varepsilon^{\mu} \partial_{\mu}\right\} \Pi_{1},
$$

where we define:

$$
\begin{gathered}
T^{a}=\int d^{10} x f^{\mathrm{abc}} A_{\mu}^{b} \frac{\delta}{\delta A_{\mu}^{a}}, \\
Y^{a}=f^{a b c} \omega^{b}\left(\omega^{c}\right)^{\dagger}
\end{gathered}
$$


In the above the following should be noted. Firstly the rotation operators $T$ and $Y$ commute with $\Pi_{1}$. Secondly we can take $\varepsilon$ outside the $\Pi_{1}$ operators because $\Delta_{0}$ commutes with $\varepsilon$. Before we evaluate $\Delta_{1}$, the following identities must be noted. They can be proved by simply writing out the relevant operators in detail and evaluating the expressions:

$$
\begin{gathered}
{\left[\partial_{\mu}, T^{a}\right]=0,} \\
{\left[\partial_{\mu}, Y^{a}\right]=0,} \\
N_{k} \partial_{\mu} \Pi_{1}=0 \text { for } k \geqq 1, \\
Y^{a}=-\left(Y^{a}\right)^{\dagger}, \\
T^{a}=-\left(T^{a}\right)^{\dagger} .
\end{gathered}
$$

Using these and other obvious properties of the various operators it is quite simple to evaluate $\Delta_{1}$, because $\Delta_{1}$ splits into several parts which are easy to understand by themselves. The first division which it is natural to make is:

$$
\Delta_{1}=\left[d_{1}+d_{1}^{\dagger}\right]^{2}=\Delta_{1}(\partial)+\Delta_{1}(T, Y) \text {. }
$$

Making use of identities like $N_{1} \partial_{\mu} \Pi_{1}=0$ and $T^{a} \Pi_{1}=\Pi_{1} T^{a}$, we have:

$$
\begin{gathered}
\Delta_{1}(\partial)=\Pi_{1}\left\{\varepsilon^{\mu} \partial_{\mu}\left[1-N_{1}\right] \partial_{\nu}^{\dagger} \varepsilon^{\nu \dagger}+\varepsilon^{\nu \dagger} \partial_{\nu}^{\dagger} \varepsilon^{\mu} \partial_{\mu}\right\} \Pi_{1} \\
=\Pi_{1}\left\{\varepsilon^{\nu \dagger} \varepsilon^{\mu}\left\{\left[\partial_{\nu}^{\dagger}, \partial_{\mu}\right]-\partial_{\mu} N_{1} \partial_{\nu}^{\dagger}\right\}+\partial_{\mu}\left[1-N_{1}\right]\left(\partial_{\mu}\right)^{\dagger}\right\} \Pi_{1}, \\
\Delta_{1}(T, Y)=\Pi_{1}\left\{-\omega^{b}\left(T^{b}+\frac{1}{2} Y^{b}\right)\left(T^{a}+\frac{1}{2} Y^{a}\right) \omega^{a \dagger}\right. \\
\left.-\left(T^{a}+\frac{1}{2} Y^{a}\right) \omega^{a \dagger} \omega^{b}\left(T^{b}+\frac{1}{2} Y^{b}\right)\right\} \Pi_{1} .
\end{gathered}
$$

At this point it is necessary to evaluate the above expression for $\Delta_{1}(\partial)$ using the full Fock representation of the various operators. The results of this are:

$$
\begin{aligned}
\Delta_{1}(\partial)= & \Pi_{1} \sum_{k=1}^{\infty}\left\{\frac{1+1 / k}{4 k !} P_{\alpha \beta \mu_{1} \cdots \mu_{k}}^{a}\left(P_{\alpha \beta \mu_{1} \cdots \mu_{k}}^{a}\right)^{\dagger}\right. \\
& \left.+\frac{1-1 / k}{4 k !} M_{\alpha \beta \mu_{1} \cdots \mu_{k}}^{a}\left(M_{\alpha \beta \mu_{1} \cdots \mu_{k}}^{a}\right)^{\dagger}\right\} \Pi_{1}
\end{aligned}
$$

where the operators $P$ and $M$ are defined by:

$$
\begin{aligned}
& P_{\alpha \beta \mu_{1} \cdots \mu_{k}}^{a}=\left(\varepsilon^{\alpha}\right)^{\dagger} A_{\beta \mu_{1} \cdots \mu_{k}}^{a}+\left(\varepsilon^{\beta}\right)^{\dagger} A_{\alpha \mu_{1} \cdots \mu_{k}}^{a}, \\
& M_{\alpha \beta \mu_{1} \cdots \mu_{k}}^{a}=\left(\varepsilon^{\alpha}\right)^{\dagger} A_{\beta \mu_{1} \cdots \mu_{k}}^{a}-\left(\varepsilon^{\beta}\right)^{\dagger} A_{\alpha \mu_{1} \cdots \mu_{k}}^{a} .
\end{aligned}
$$

The evaluation of $\Delta_{1}(T, Y)$ uses only obvious properties of the operators $T$ and $Y$ and the result is:

$$
\Delta_{1}(T, Y)=\Pi_{1}\left\{\left(T^{a}+\frac{1}{2} Y^{a}\right)\left(T^{a}+\frac{1}{2} Y^{a}\right)^{\dagger}+\frac{1}{4}\left(Y^{a}\right)\left(Y^{a}\right)^{\dagger}\right\} \Pi_{1} .
$$




\section{The Space $E_{2}$}

All of these terms are manifestly positive semidefinite of the form $Q_{i} Q_{i}^{\dagger}$. Hence we can deduce the following equations for $E_{2}$ :

$$
\begin{gathered}
\varepsilon^{\alpha}\left(A_{\beta \mu_{1} \cdots \mu_{k}}^{a}\right)^{\dagger} E_{2}=0 \text { for } k \geqq 2, \\
{\left[\varepsilon^{\alpha}\left(A_{\beta \mu}^{\prime a}\right)^{\dagger}+\varepsilon^{\beta}\left(A_{\alpha \mu}^{\prime a}\right)^{\dagger}\right] E_{2}=0,} \\
Y^{a} E_{2}=0 \\
T^{a} E_{2}=0 \\
{\left[1-N_{1}\right]\left(\partial_{\mu}\right)^{\dagger} E_{2}=0}
\end{gathered}
$$

where we use the notation of Eq. (34) and use the abbreviation:

$$
A_{\alpha \beta}^{\prime}=-A_{\beta \alpha}^{\prime}=A_{a, \beta}^{\prime}=-A_{\beta, \alpha}^{\prime} \text {. }
$$

Most of these equations are easily recognized and solved. Thus it is evident that the $Y$ and $T$ equations are simply the requirement that $E_{2}$ be invariant under rigid rotations in the Lie algebra; performed separately on $\omega$ and on $A$. These equations bring in the classical theory of the cohomology of Lie algebras: [23, 6-8, 21]. Equation (107) indicates that any term in $E_{2}$ that is not multiplied by $\varepsilon^{10}$ defined in Eq. (75) must be independent of the fields $A_{\mu, \mu_{1} \cdots \mu_{k}}^{a}$ for $k \geqq 2$ but may depend on $\omega$ (without derivatives). Equation (108) has non-trivial solutions which are discussed in Appendix $\mathrm{C}$ and described below. Evidently any polynomial of degree higher than ten in $\varepsilon$ must be zero in ten spacetime dimensions. Putting these observations together means that $E_{2}$ must be the sum of two kinds of terms which we can call normal $(n)$ and exceptional $(e)$. It has the form:

$$
E_{2}=\sum_{i, j} n_{i J} I_{l}\left(A_{\alpha, \mu_{1} \cdots \mu_{k}}^{\prime a}\right) T_{J}(\omega) \varepsilon^{10}+\sum_{i, j} e_{i J} I_{i}^{\prime}\left(A_{\alpha \beta}^{\prime a}, \varepsilon\right) T_{j}(\omega)
$$

where $n$ and $e$ are numerical coefficients and $I, I^{\prime}$ and $T$ are polynomials which are invariant under the gauge rotations of the variables indicated as their arguments. These objects must satisfy the following equations:

$$
\begin{gathered}
Y^{a} T_{l}(\omega)=0, \\
T^{a} I_{l}\left(A^{\prime}\right)=0, \\
T^{a} I_{l}^{\prime}\left(A_{\alpha \beta}^{\prime}, \varepsilon\right)=0, \\
\left(\mathrm{~A}_{\alpha, \mu_{1} \cdots \mu_{k}}^{a}\right)^{\dagger} I_{i}^{\prime}\left(A_{\alpha \beta}^{\prime}, \varepsilon\right) \text { for } k \geqq 2, \\
{\left[\varepsilon^{\alpha}\left(A_{\beta \mu}^{\prime a}\right)^{\dagger}+\varepsilon^{\beta}\left(A_{\alpha \mu}^{\prime a}\right)^{\dagger}\right] I_{i}^{\prime}\left(A_{\alpha \beta}^{\prime}, \varepsilon\right)=0,} \\
{\left[1-N_{1}\right] \partial_{\mu}^{\dagger} I_{l}\left(A^{\prime}\right) .}
\end{gathered}
$$

The solution of Eq. (118) is straightforward and is discussed in Appendix C. The basic solution of (118) is the term:

$$
F^{a}=A_{\mu \nu}^{a} \varepsilon^{\mu} \varepsilon^{\nu}
$$


and all other solutions are formed by taking products of this with itself and with free $\varepsilon$ 's:

$$
I^{\prime}\left(A_{\mu \nu}^{\prime}, \varepsilon\right)=F^{a_{1}} F^{a_{2}} \cdots F^{a_{t}} t^{a_{1} a_{2} \cdots a_{t}} \varepsilon_{\nu_{1}} \varepsilon_{\nu_{2}} \cdots \varepsilon_{\nu_{1}} .
$$

in this formula, $t$ is a symmetric tensor which is invariant under the Lie algebra and the terms

$$
\varepsilon_{v_{1}} \varepsilon_{v_{2}} \cdots \varepsilon_{v_{\mathrm{r}}}
$$

introduce free Lorentz indices into the problem. The total number of variables $\varepsilon$ in the expression is restricted to be less than or equal to the dimension $D$ of spacetime which for our present purposes is $10 . E_{2}$ can now be written in the form:

$$
\begin{aligned}
E_{2}= & \sum_{i, j} n_{i j} I_{i}\left(A^{\prime}\right) T_{j}(\omega) \varepsilon^{10} \\
& +\sum_{k, r, j} e_{k, r, j} F^{a_{1}} F^{a_{2}} \cdots F^{a_{k}} t^{a_{1} a_{2} \cdots a_{k}} \varepsilon_{v_{1}} \varepsilon_{v_{2}} \cdots \varepsilon_{v_{r}} T_{j}(\omega),
\end{aligned}
$$

where the number of $e_{k, r, j}$ type terms is restricted by the fact that a product of eleven or more $\varepsilon$ 's is zero in ten dimensions. These expressions are meant to include the degenerate case when one of the indicated expressions is just 1 . There is some overlap when the number of $\varepsilon$ 's is ten between the $n$ and the e terms. This overlap should be resolved by placing all the duplicated terms in the exceptional part, so that they can be eliminated in the higher orders of $E_{r}$ as discussed below.

\section{The Operators $d_{r}$ and the Spaces $E_{r}$ for $r \geqq 2$}

There are still nonzero operators $d_{r}$ with $r \geqq 2$ that are non-zero, but they act in a very simple way to eliminate some very specific parts of $E_{2}$ from $E_{\infty}$. All of the eliminated vectors are of the exceptional (e) type. The normal (n) part of $E_{2}$ is not affected by $d_{r}$ for $r \geqq 2$, because Eq. 52 cannot be satisfied given the fact that all primitive $T(\omega)$ have an odd number of $\omega$ in them, as discussed below. The effect of these $d_{r}$ on the exceptional vectors is most easily seen from the following "Spectral Sequence Diagram:"

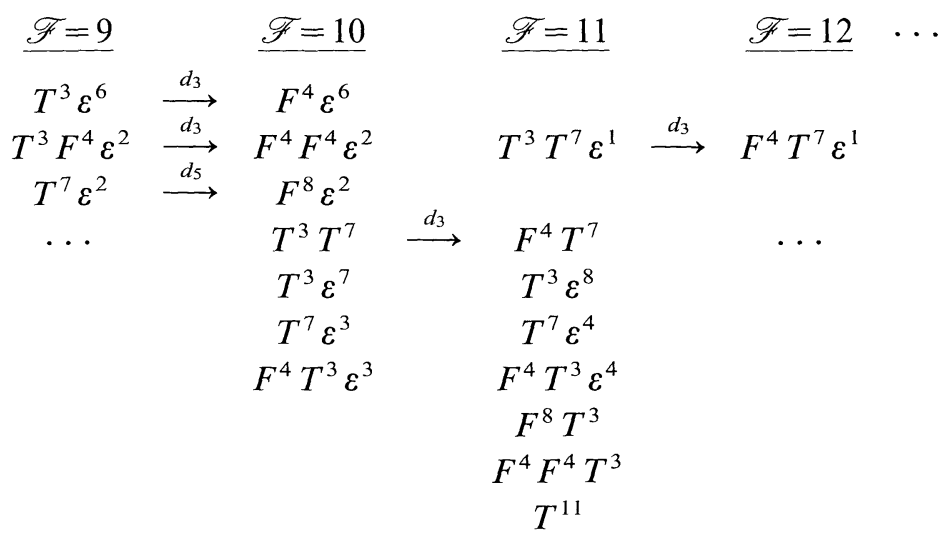


where we use the notation

$$
T^{3}=f^{a b c} \omega^{a} \omega^{b} \omega^{c} .
$$

Similarly $T^{7}$ and $T^{11}$ are defined to be contractions with the indicated number of $\omega$ fields. The objects $F$ are defined by:

$$
\begin{gathered}
F^{4}=A_{\mu \nu}^{a} \varepsilon^{\mu} \varepsilon^{\nu} A_{\lambda \sigma}^{a} \varepsilon^{\lambda} \varepsilon^{\sigma}, \\
F^{8}=A_{\mu \nu}^{a} \varepsilon^{\mu} \varepsilon^{\nu} A_{\lambda \sigma}^{b} \varepsilon^{\lambda} \varepsilon^{\sigma} A_{\kappa \eta}^{c} \varepsilon^{\kappa} \varepsilon^{\eta} A_{\zeta \zeta}^{d} \varepsilon^{\zeta} \varepsilon^{\xi} t^{a b c d},
\end{gathered}
$$

where $t^{a b c d}$ is the totally symmetric invariant tensor with four indices that exists in $S O(32)$ according to the results below. The notation $\varepsilon^{i}$ is shorthand for the expressions of the form:

$$
\varepsilon^{i}=\varepsilon_{\mu_{1}} \varepsilon_{\mu 2} \cdots \varepsilon_{\mu_{i}}
$$

where the Lorentz indices are free (i.e. uncontracted).

A brief discussion of the Lie algebra cohomology of $S O(32)$ is in order at this point. The set of all $T(\omega)$ satisfying Eq. 114 is generated by a set of primitive $T(\omega)$ by multiplication. The primitive $T(\omega)$ can be constructed using the primitive invariant totally antisymmetric tensors of the Lie algebra and the classical result [23], (Vol. 3, p. 259) is that the Poincaré polynomial is given by the following for $m=16$ :

$$
P_{2 m}(t)=\left[1+t^{2 m-1}\right] \prod_{p=1}^{m-1}\left[1+t^{4 p-1}\right]
$$

and the Poincaré series is given by:

$$
S_{2 m}(t)=\left[1-t^{2 m}\right] \prod_{p=1}^{m-1}\left[1-t^{4 p}\right]^{-1} .
$$

The coefficient of $t^{j}$ in the Poincare polynomial is the number of linearly independent invariant antisymmetric tensors with $j$ indices, and the coefficient of $t^{2 J}$ in the Poincare series is the number of linearly independent invariant symmetric tensors with $j$ indices. Hence we see that in $S O(32)$ there is one primitive totally antisymmetric tensor with $3,7,11 \cdots 59$ indices and also an extra one with 31 indices. And there are primitive totally symmetric invariant tensors with 2, 4, $6 \cdots 30$ indices and also an extra one with 16 indices. We can also deduce from these expressions that the totally antisymmetrized product of any number of nonidentical primitive tensors does not vanish, so that for example we know that $T^{3} T^{7}$ does not vanish. There is a relation of the following form between these two sets of tensors:

$$
\begin{aligned}
& \operatorname{Symm}\left\{T^{a_{1} a_{2} \cdots a_{2 p-2} b_{p}} f^{b_{1} a_{1} a_{2}} f^{b_{2} a_{3} a_{4}} \cdots f^{b_{p-1} a_{2 p-3} a_{2 p-2}}\right\} \\
& \quad=\text { const }\left\{t^{b_{1} b_{2} \cdots b_{p}}\right\}
\end{aligned}
$$

where "Symm" means "Symmetrize over $b_{1} \cdots b_{p}$ " and the constant on the right is not zero. Here $T$ is the primitive totally antisymmetric tensor with $2 p-1$ indices and $t$ is the primitive totally symmetric tensor with $p$ indices. There are two antisymmetric tensors with 31 indices that correspond with the two symmetric 
tensors with 16 indices in this formula. Conversely one also has the formula:

$$
\begin{aligned}
& \text { Antisym }\left\{t^{a_{1} a_{2} \cdots a_{p-1} b_{2 p-1}} f^{a_{1} b_{1} b_{2}} f^{a_{2} b_{3} b_{4}} \cdots f^{a_{p-1} b_{2 p}-3 b_{2}-2}\right\} \\
& \quad=\text { const } T^{b_{1} b_{2} \cdots b_{2 p-1}} .
\end{aligned}
$$

These identities play an important role in the diagram. A proof of them can actually be obtained from the present formalism by noting that there can be no invariants in $H_{\mathscr{T}}$ with negative ghost charge $\mathscr{G}$ [defined in Eq. (54) above], so that the vectors in $E_{2}$ with $\mathscr{F}<D$ must be eliminated using these identities, given the form (see below) of the operators $d_{r}$. This also requires the knowledge that the isomorphism $E_{\infty} \rightarrow H_{\mathscr{T}}$ converts an expression in $E_{\infty}$ with $\mathscr{F}=g$ into an expression in $H_{\mathscr{T}}$ with $\mathscr{G}=g-D$, which is explained in Appendix B.

The upper index on the abbreviated forms $T^{3}, F^{4}$ and $\varepsilon^{2}$, etc. corresponds to the eigenvalue of the operator $\mathscr{F}$ of Eq. (55) for the corresponding object. Hence one can see that in the diagram every object under a given value of $\mathscr{F}$ has indices which sum to that $\mathscr{F}$.

The diagram contains all the objects that can be constructed in the exceptional sector with the given values of $\mathscr{F}$ in the theory. The lines joining various pairs of objects represent the action of the operators $d_{3}$ and $d_{5}$. If two objects are joined by a line, then they do not survive to $E_{\infty}$, and if they are not joined by a line, then they do survive to $E_{\infty}$. We have cut off the diagram at values of $\mathscr{F}$ less than 9 and greater than 12, and the polynomials with $\mathscr{F}=9,12$ are only shown insofar as they affect the terms with $\mathscr{F}=10,11$. Of course, the diagram could be extended to include them.

The operator $d_{3}$ is given by:

$$
d_{3}=\Pi_{2} \varepsilon^{\mu} \varepsilon^{\nu} A_{\mu \nu}^{a} \varepsilon^{\lambda} \varepsilon^{\sigma} A_{\lambda \sigma}^{b} \omega^{a \dagger} f^{b c d} \omega^{b \dagger} \omega^{c \dagger} \Pi_{2},
$$

as can be seen using Eq. (63) and using the properties of $\Pi_{3}=\Pi_{2}$ [it is first necessary to note that $d_{2}=0$, using Eq. (62) and the properties of $\left.\Pi_{2}\right]$. The operator $d_{3}$ takes $T^{3}$ into $F^{4}$, and its adjoint takes $F^{4}$ into $T^{3}$, using relations of the form in Eq. (131) and (132). Similarly $d_{5}$ takes $T^{7}$ into $F^{8}$ and its adjoint takes $F^{8}$ into $T^{7}$.

Note that the following objects are not connected by a line to anything else:

$$
\begin{gathered}
\mathscr{F}=10: T^{3} \varepsilon^{7}, T^{7} \varepsilon^{3}, F^{4} T^{3} \varepsilon^{3}, \\
\mathscr{F}=11: T^{3} \varepsilon^{8}, T^{7} \varepsilon^{4}, F^{4} T^{3} \varepsilon^{4}, F^{8} T^{3}, F^{4} F^{4} T^{3}, T^{11} .
\end{gathered}
$$

It is easy to see by considering 51 and 52 that these objects must survive to $E_{\infty}$. For example, there is nothing left in the $\mathscr{F}=9,11$ sectors that has the right value of $N$ to connect with the objects in the $\mathscr{F}=10$ by a $d_{r}$ for $r>5$. For this purpose we note that:

$$
\begin{gathered}
N F^{4}=6 F^{4}, \\
N F^{8}=12 F^{8}, \\
N T^{i}=i T^{\imath}, \\
N \varepsilon^{i}=i \varepsilon^{l} .
\end{gathered}
$$

Consequently, when we have written down all the objects with a given value of $\mathscr{F}$ and have used all the $d_{r}$ that do not require too large a value of $N$ for that 
value of $\mathscr{F}$, we know that those terms can not be eliminated by any higher $d_{r}$. The diagram shows that $d_{5}$ is all that is necessary to get all the information about $\mathscr{F}$ up to 11 in this case.

\section{The Spaces $E_{\infty}$ and $H$}

Now let us examine $E_{\infty}$. It consists of a large set of normal objects with $\mathscr{F}=10$ plus the $\mathscr{G}$ of the $T^{j}$ object plus a small set of exceptional objects with $\mathscr{F}=10$, plus a small set of exceptional objects with $\mathscr{F}=11$, plus objects with higher values of $\mathscr{F}$ that we have not bothered to deal with. The correspondence between $H$ and $E_{\infty}$ is easy to see. For the first kinds of terms one simply uses $I\left(A^{\prime}\right)$ as the lowest term in a series in $A$ for the relevant object in $H$, and substitutes:

$$
\varepsilon^{10} \rightarrow \int d^{10} x
$$

For the exceptional terms a little more work is needed. See Appendix B for a short discussion of this isomorphism. For example the object

$$
T^{7} \varepsilon^{4} \in E_{\infty} \rightarrow \int d^{10} x\left[\varepsilon^{\mu \nu \lambda \sigma \mu_{1} \cdots \mu_{6}} A_{\mu_{1} \mu_{2}}^{a} A_{\mu_{3} \mu_{4}}^{b} A_{\mu_{5} \mu_{6}}^{c} \omega^{d} t^{a b c d}+\cdots\right] \in H .
$$

The form of the higher order terms indicated by ... can be worked out by analogy with the well known methods for constructing the full expression of the usual anomalies [34]. It could also be constructed with the Noether method (see Appendix B).

This expression is like the well known expressions for Lorentz invariant anomalies except that it has dimension -4 in the units where the action has dimension -6 and the usual anomaly has dimension 0 , and it also has four free Lorentz indices.

\section{Part IV. Conclusion}

\section{Polynomials in $E_{\infty}$}

First of all let us summarize the results obtained for the specific example that we have worked out in the foregoing.

Theorem 3. The ghost charge zero sector of $H_{\mathscr{T}}$ for pure Yang-Mills theory in ten dimensions with gauge group $S O(32)$ is isomorphic to the $\mathscr{F}=10$ sector of $E_{\infty}$, which has the form:

$$
E_{\infty}^{\mathscr{F}=10}=\sum_{i} n_{i} I_{\imath}\left(A_{\alpha, \mu_{1} \ldots \mu_{k}}^{\prime a}\right) \varepsilon^{10}+e_{1} T^{3} \varepsilon^{7}+e_{2} T^{7} \varepsilon^{3}+e_{3} F^{4} T^{3} \varepsilon^{3} .
$$

where $n_{t}$ and $e_{t}$ are numerical coefficients and the rest of the notation is defined above.

Theorem 4. The ghost charge one sector of $H_{\mathscr{T}}$ for pure Yang-Mills theory in ten dimensions with gauge group $S O(32)$ is isomorphic to the $\mathscr{F}=11$ sector of $E_{\infty}$, which has the form: 


$$
\begin{aligned}
E_{\infty}^{\mathscr{F}=11}= & e_{1} T^{3} \varepsilon^{8}+e_{2} T^{7} \varepsilon^{4}+e_{3} F^{4} T^{3} \varepsilon^{4}+e_{4} F^{8} T^{3} \\
& +e_{5} F^{4} F^{4} T^{3}+e_{6} T^{11}
\end{aligned}
$$

where again $e_{1}$ are numerical coefficients and the rest of the notation is defined above.

Let us now make a general statement valid for all groups and dimensions of spacetime.

Theorem 5. To compute the BRS cohomology of a pure Yang-Mills theory, one must first specify the dimension of spacetime $D$, the gauge group, and the ghost charge $g$, where $\mathscr{F}=\mathscr{G}+D=g+D$. Then one uses the isomorphism $E_{\infty} \approx H$. There are two kinds of solutions in $E_{\infty}$. The normal solutions in $E_{\infty}$ take the form

$$
E_{\infty}^{\mathscr{F}=g+D}=\sum_{i} n_{i} I_{l}\left(A_{\alpha, \mu_{1} \cdots \mu_{k}}^{\prime a}\right) T^{g} \varepsilon^{D}
$$

The exceptional solutions are found by writing down all the terms of the form

$$
F^{a_{1}} F^{a_{2}} \cdots F^{a_{k}} t^{a_{1} a_{2} \cdots a_{k}} \varepsilon_{v_{1}} \varepsilon_{v_{2}} \cdots \varepsilon_{v_{r}} T^{j}(\omega)
$$

(from (123)) which have $\mathscr{F}=g+D$. Next one picks out the terms of the same form with $\mathscr{F}=g+D-1$ and $\mathscr{F}=g+D+1$ that can be connected with the terms with $\mathscr{F}=g+D$ by operators $d_{r}$ and that are not eliminated earlier by lower $d_{r}$. One does this until inspection of the $N$ eigenvalues shows that no further $d_{r}$ can be relevant, using relation 51 . The normal and the remaining exceptional vectors are the relevant polynomials in $E_{\infty}$ and one proceeds from them to $H$ in the method explained in Appendix $B$.

The demonstrations of all these results are contained in the foregoing sections.

Now let us make the isomorphism $E_{\infty} \rightarrow H_{\mathscr{T}}$ explicit using the methods described in Appendix B.

\subsection{Ghost Charge Zero}

The cohomology space for $G=0$ for $S O$ (32) Yang-Mills theory in ten dimensions is spanned by polynomials lying in two sectors which we call normal and exceptional:

1. Normal Sector: This is independent of the group and dimension of space-time except in obvious ways. To any non-zero polynomials constructed from the fields $A^{\prime}$ defined in Eq. (34) and (31) and fully contracted in the group indices with invariant tensors of the Lie algebra, there exists a corresponding polynomial in the normal sector, and it can be constructed by adding terms of higher order in the coupling constant to the starting polynomial in the well known Noether way.

2. Exceptional Sector: For the case of $S O$ (32) Yang-Mills in ten dimensions, this consists of the following three objects:

$$
\begin{gathered}
\int d^{10} x \varepsilon^{\alpha \beta \gamma \delta \varepsilon \zeta \eta \mu_{1} \mu_{2} \mu_{3}}\left\{\partial_{\mu_{1}} A_{\mu_{1}}^{a} A_{\mu_{3}}^{a}\right\}, \\
\int d^{10} x \varepsilon^{\left.\alpha \beta \gamma \mu_{1} \cdots \mu\right\urcorner}\left\{\partial_{\mu_{1}} A_{\mu_{2}}^{a} \partial_{\mu_{3}} A_{\mu_{4}}^{b} \partial_{\mu_{5}} A_{\mu_{6}}^{c} A_{\mu_{7}}^{d} t^{a b c d}+\cdots\right\}, \\
\int d^{10} x \varepsilon^{\alpha \beta \gamma \mu_{1} \cdots \mu_{7}}\left\{\partial_{\mu_{1}} A_{\mu_{2}}^{a} \partial_{\mu_{3}} A_{\mu_{4}}^{a} \partial_{\mu_{5}} A_{\mu_{6}}^{b} A_{\mu_{7}}^{b}+\cdots\right\},
\end{gathered}
$$


where the notation indicates that terms with more $A$ 's need to be added using the Noether procedure. The dimensions and number of free Lorentz indices of these objects are $(\operatorname{dim}$, lor $)=(-7,7),(-3,3)$, and $(-3,3)$ respectively.

\subsection{Ghost Charge One}

The cohomology space for $G=1$ for $S O$ (32) Yang-Mills theory in ten dimensions consists of the following six polynomials (which are all in the exceptional sector) [from Eq. (135)]:

$$
\begin{gathered}
\int d^{10} x \varepsilon^{\alpha \beta \mu_{1} \cdots \mu_{8}}\left\{\partial_{\alpha} A_{\beta}^{a} \omega^{a}+\cdots\right\} \\
\int d^{10} x \varepsilon^{\alpha \beta \gamma \delta \mu_{1} \cdots \mu_{6}}\left\{\partial_{\mu_{1}} A_{\mu_{2}}^{a} \partial_{\mu_{3}} A_{\mu_{4}}^{b} \partial_{\mu_{5}} A_{\mu_{6}}^{c} \omega^{d} t^{a b c d}+\cdots\right\}, \\
\int d^{10} x \varepsilon^{\alpha \beta \gamma \delta \mu_{1} \cdots \mu_{6}}\left\{\partial_{\mu_{1}} A_{\mu_{2}}^{a} \partial_{\mu_{3}} A_{\mu_{4}}^{a} \partial_{\mu_{5}} A_{\mu_{6}}^{b} \omega^{b}+\cdots\right\} \\
\int d^{10} x \varepsilon^{\mu_{1} \cdots \mu_{10}}\left\{\partial_{\mu_{1}} A_{\mu_{2}}^{a} \partial_{\mu_{3}} A_{\mu_{4}}^{b} \partial_{\mu_{5}} A_{\mu_{6}}^{c} \partial_{\mu_{7}} A_{\mu_{8}}^{d} \partial_{\mu_{9}} A_{\mu_{10}}^{e} \omega^{f} t^{a b c d e f}+\cdots\right\} \\
\int d^{10} x \varepsilon^{\mu_{1} \cdots \mu_{10}}\left\{\partial_{\mu_{1}} A_{\mu_{2}}^{a} \partial_{\mu_{3}} A_{\mu_{4}}^{b} \partial_{\mu_{5}} A_{\mu_{6}}^{c} \partial_{\mu_{7}} A_{\mu_{8}}^{d} \partial_{\mu_{9}} A_{\mu_{10}}^{e} \omega^{e} t^{a b c d}+\cdots\right\} \\
\int d^{10} x \varepsilon^{\mu_{1} \cdots \mu_{10}}\left\{\partial_{\mu_{1}} A_{\mu_{2}}^{a} \partial_{\mu_{3}} A_{\mu_{4}}^{a} \partial_{\mu_{5}} A_{\mu_{6}}^{b} \partial_{\mu_{7}} A_{\mu_{8}}^{b} \partial_{\mu_{9}} A_{\mu_{10}}^{c} \omega^{c}+\cdots\right\}
\end{gathered}
$$

The dimensions and number of free Lorentz indices of these objects are $(\operatorname{dim}$, lor $)=(-8,8),(-4,4),(-4,4),(0,0),(0,0)$, and $(0,0)$ respectively.

Clearly it would be straightforward to generalize these results to other groups and numbers of dimensions of spacetime. A natural question that arises is whether all or only some of these objects have any interpretation in terms of anomalies of the corresponding theories.

\section{Discussion}

Using the general results of Brandt et al. [10-13], it should be possible to generate general formulae for the case of the BRS operator in Yang-Mills theory which would include all dimensions and compact groups and would also generate nonLorentz invariant polynomials in the cohomology space. Alternatively, one could simply draw a diagram like 124 and eliminate the forms that do not survive to $E_{\infty}$ using the various $d_{r}$ that could be formed for the given group and spacetime dimensions. In many ways the use of the general formula and the use of the diagram must be quite similar if both yield the same rather complicated result.

Since the argument here is complicated and the arguments of Brandt et al. are also not simple, it is encouraging that, for the specific case examined here, the general formula of Brandt et al. gives the same result as that obtained here for the Lorentz-invariant part of the ghost charge one sector. Their analysis (see Formula (27) of [10]) gives the same number of terms (three) for the Lorentzinvariant part of 143 as that obtained here.

In the previous section we have listed the various objects that are found in the cohomology space $H$ of the restricted BRS operator of $S O$ (32) Yang Mills theory in 10 dimensions for ghost charge zero and one. The polynomials with free Lorentz indices are closely related to objects in other dimensions of spacetime without free Lorentz indices, just as the polynomials with nonzero ghost charge 
are related to polynomials in $H$ with zero ghost charge in other dimensions. The present rather laborious method using the higher operators $d_{3}$ and $d_{5}$ has the advantage (and the disadvantage) that it does not require insight into the various known methods of constructing polynomials in $H$ by reducing or increasing dimension.

In a sense, the present method is a "stupid" and mechanical method. It does not naturally give general results. It is better adapted to analysis of one specifc problem at a time, although of course the analysis of a number of such problems would lead to suitable general formulae.

This rather mechanical feature does however render the method useful for the supersymmetric theories where dimensional reduction techniques are likely to be much harder to find, since typically supersymmetric theories, unlike purely bosonic theories, undergo severe transformations (or disappear altogether) when the dimension of spacetime is changed. This feature of supersymmetric theories might render techniques that are based on dimensional reduction difficult to apply.

On the other hand, the unrestricted problem for all theories (i.e. including sources for the gauge variation, etc.) including pure Yang-Mills is still outstanding. The present method is arduous for this case. This problem appears to be a particularly important one for supersymmetric Yang Mills theory in ten dimensions since there is probably no restricted operator in that case.

Finally some remarks on the examination of non-Lorentz invariant elements of $H$ are in order. It might be thought at first that these are of little interest, because they will not eliminate theories, since the action is Lorentz invariant. These elements of the cohomology space are relevant only if one wants to analyze the renormalization of non-Lorentz invariant composite operators. But surely this is a reasonable thing to do in theories where these operators are the interpolating local fields for bound states with the appropriate quantum numbers. For example the proton is believed to be a gauge invariant spinor bound state in a Yang-Mills theory of quarks and gluons. As mentioned above, in supersymmetric theories [18], it appears that there can be anomalies in the renormalization of gauge-invariant spinor composite fields. It is possible that such anomalies may have important physical consequences.

\section{Part V. Appendices}

\section{A. Construction of $d_{r}$}

The spaces $E_{r}^{p}$ are easily analyzed for low $r$. For $r=0$ we see from the definition in Eq. (49) that:

$$
E_{0}^{p}=P_{p} \cap\left(\delta F_{p+1}\right)^{\perp} \cap\left(\delta^{\dagger} F^{p-1}\right)^{\perp}=P_{p}
$$

where we use the relations:

and the relation:

$$
\begin{gathered}
\delta F_{p+1} \subset F_{p+1} \subset F_{p}, \\
\delta^{\dagger} F^{p-1} \subset F^{p-1} \subset F^{p},
\end{gathered}
$$

$$
M \subset N \Leftrightarrow N^{\perp} \subset M^{\perp}
$$


valid for any subspaces $M$ and $N$, along with the evident relations such as:

It follows that:

$$
\left(F_{p+1}\right)^{\perp}=F^{p} \text {. }
$$

$$
E_{0}=\sum_{p} P_{p}=P
$$

so that $E_{0}$ is simply the whole space. Next consider $E_{1}$ :

$$
E_{1}^{p}=P_{p} \cap\left(\delta F_{p}\right)^{\perp} \cap\left(\delta^{\dagger} F^{p}\right)^{\perp} .
$$

Clearly, if $x$ is a vector in $E_{1}^{p}$, it satisfies the relations:

$$
\left\langle x \mid \delta F^{p}\right\rangle=\left\langle\delta^{\dagger} x \mid F^{p}\right\rangle=\left\langle\delta_{0}^{\dagger} x \mid P_{p}\right\rangle=0
$$

and a similar set of equations involving the adjoints, and so $x$ satisfies the relations:

$$
\delta_{0}^{\dagger} x=\delta_{0} x=0
$$

and so clearly we can write

$$
E_{1}^{p}=P_{p} \cap \operatorname{Ker} \delta_{0} \cap \operatorname{Ker} \delta_{0}^{\dagger},
$$

where $d_{0}=\delta_{0}$ has been used. For $r=2$, we have:

$$
E_{2}^{p}=E_{1}^{p} \cap\left(\delta F_{p}^{-1} \cap F_{p}\right)^{\perp} \cap\left(\delta^{\dagger} F^{p+1} \cap F^{p}\right)^{\perp} .
$$

To proceed further we first note the following identity:

$$
\left(\delta F_{p-1} \cap F_{p}\right)=\delta\left(E_{1}^{p}+F_{p}\right) .
$$

To prove this, suppose that $x$ is a vector of the form on the left side of this equation. Then it can be written:

$$
x=\left[\delta_{0} y_{p}+\delta_{1} y_{p-1}\right]+\left[\delta_{0} y_{p+1}+\delta_{1} y_{p}+\delta_{2} y_{p-1}\right]+\cdots,
$$

where the vectors $y_{p}$ are in the subspaces $P_{p}$ and the vector $y_{p-1}$ is subject to the constraint:

$$
\delta_{0} y_{p-1}=0
$$

But this constraint implies that:

$$
y_{p-1}=\delta_{0} z_{p-1}+e_{p-1}
$$

for some vector $z_{p-1} \in P_{p-1}$ and some vector $e_{p-1} \in E_{1}^{p-1}$ defined in Eq. (164). Hence we see that $x$ can be written in the form:

$$
x=\delta\left[e_{p-1}+\delta_{0} z_{p-1}+y_{p}+y_{p+1}+\cdots\right],
$$

which proves the relation, since it is evident that all vectors on the right in Eq. (166) are also included on the left. A similar relation can of course be demonstrated for the adjoints. Using these and keeping track of the eigenvalues of $N$ quickly yields the relation: 


$$
E_{2}^{p}=E_{1}^{p} \cap\left(\delta_{1} E_{1}^{p-1}\right)^{\perp} \cap\left(\delta_{1}^{\dagger} E_{1}^{p+1}\right)^{\perp},
$$

which can be written in the form:

where

$$
E_{2}^{p}=E_{1}^{p} \cap \operatorname{Ker} d_{1} \cap \operatorname{Ker} d_{1}^{\dagger}
$$

$$
d_{1}=\Pi_{1} \delta_{1} \Pi_{1}
$$

with $\Pi_{1}$ the orthogonal projection operator onto $E_{1}$. The derivation of the higher operators $d_{r}$ is a straightforward exercise along the same lines as these. It rapidly becomes quite involved in the general case. In practice the operators are easy to guess in most cases, which is also what happens in the applications that are documented in the mathematical literature.

\section{B. The Noether Procedure and the Isomorphism $E_{\infty} \rightarrow H$}

The isomorphism $E_{\infty} \rightarrow H$ is closely related to the procedure widely known as the Noether method for building up invariants [41]. The correspondence works as follows: If $e \in E_{\infty}$, and $\delta=\delta_{0}+\delta_{1}$ as usual, with $\left[N, \delta_{i}\right]=i \delta_{i}$, where $N$ generates the spectral sequences, then it follows that:

$$
\begin{aligned}
\Delta_{1} e & =0 \Rightarrow \Pi_{1}\left[\delta_{1}+\delta_{1}^{\dagger}\right] e=0 \\
& \Rightarrow\left[1-\Pi_{1}\right]\left[\delta_{1}+\delta_{1}^{\dagger}\right] e=\left[\delta_{1}+\delta_{1}^{\dagger}\right] e=\Delta_{0} \frac{1}{\Delta_{0}}\left[\delta_{1}+\delta_{1}^{\dagger}\right] e
\end{aligned}
$$

and the last equation can be written in the form:

$$
\left[\delta_{1}+\delta_{1}^{\dagger}\right] e=\left[\delta_{0}+\delta_{0}^{\dagger}\right] e^{\prime},
$$

where $e^{\prime}$ is defined by:

$$
e^{\prime}=\left[\delta_{0}+\delta_{0}^{\dagger}\right] \frac{1}{\Delta_{0}}\left[\delta_{1}+\delta_{1}^{\dagger}\right] e,
$$

and hence we have:

$$
\left[\delta+\delta^{\dagger}\right]\left[e-e^{\prime}\right]=-\left[\delta_{1}+\delta_{1}^{\dagger}\right] e^{\prime}
$$

Now we can find an $e^{\prime \prime}$ to cancel the term on the right using the relation:

$$
d_{2} e=0 \Rightarrow \Pi_{1} \delta_{1} \frac{\delta_{0}^{\dagger}}{\Delta_{0}} \delta_{1} e=0
$$

and also its adjoint. In this way, it is, no doubt, possible to build up an element $v=e-e^{\prime}+\cdots$ which is in the cohomology space of the operator $\Delta=\left(\delta+\delta^{\dagger}\right)^{2}$. Then one would keep only the terms with $\varepsilon^{D}$ in them and use the substitution 140 to generate an element $\int d^{D} x v^{\prime} \in H_{\mathscr{T}}$ that corresponds to the original element $e \in E_{\infty}$. Note that it follows that the isomorphism $E_{\infty} \rightarrow H_{\mathscr{T}}$ converts an expression in $E_{\infty}$ with $\mathscr{F}=g$ into an expression in $H_{\mathscr{T}}$ with $\mathscr{G}=g-D$. 
In practice it seems simpler to just work directly from a general expression with the right dimension and other quantum numbers that are preserved by the isomorphism and find the element $\boldsymbol{v}^{\prime}$ directly.

\section{Solution of the Exceptional Equations}

This appendix is devoted to a demonstration of the very important and simple theorem, which is used in Sect. 9.

Theorem 6. All solutions of the equations:

$$
\left[\varepsilon^{\alpha}\left(A_{\beta \mu}^{\prime a}\right)^{\dagger}+\varepsilon^{\beta}\left(A_{\alpha \mu}^{\prime a}\right)^{\dagger}\right] P=0
$$

are of the form:

$$
P=I^{\prime}\left(A_{\mu \nu}^{\prime}, \varepsilon\right)=F^{a_{1}} F^{a_{2}} \cdots F^{a_{t}} t^{a_{1} a_{2} \cdots a_{t}} \varepsilon_{\nu_{1}} \varepsilon_{\nu_{2}} \cdots \varepsilon_{\nu_{r}}+\varepsilon^{D} Y,
$$

where

$$
F^{a}=A_{\mu \nu}^{a} \varepsilon^{\mu} \varepsilon^{\nu} .
$$

We have included a term $Y$ which is unconstrained since it is multiplied by the tensor $\varepsilon^{D}$ defined in Eq. (128), where $D$ is the dimension of spacetime, which means that $\varepsilon^{D+1}=0$, which in turn implies that Eq. (179) is trivially satisfied for this case.

In this formula, $t$ is a symmetric tensor. In order to satisfy the equation $T^{a} P=0$, it should be an invariant under the Lie algebra. The terms:

$$
\varepsilon_{v_{1}} \varepsilon_{v_{2}} \cdots \varepsilon_{v_{r}}
$$

introduce free Lorentz indices into the problem. The total number of variables $\varepsilon$ in the expression is restricted to be less than or equal to the dimension $D$ of spacetime.

Proof. Denote $\left(A_{\beta \mu}^{\prime a}\right)^{\dagger} P=P^{a \beta \mu}$. Then we have the equation:

$$
\varepsilon_{\alpha} P_{\beta \mu}^{a}=-\varepsilon_{\beta} P_{\alpha \mu}^{a}
$$

and the equation

$$
P_{\beta \mu}^{a}=-P_{\mu \beta}^{a}
$$

It follows that $\varepsilon_{\alpha} P_{\beta \gamma}^{a}$ is totally antisymmetric in the indices $\alpha, \beta$, and $\gamma$. Furthermore this antisymmetriy must be an identity in the form of the variables not merely an equation that can be satisfied for some particular "values" (a dubious term when speaking of anticommuting quantities like $\varepsilon$ ) of the variables $\varepsilon_{\alpha}$ and $P_{\beta \gamma}^{a}$. The only expression containing at least $\varepsilon_{\alpha}$ that has three indices and is identically antisymmetric is $\varepsilon_{\alpha} \varepsilon_{\beta} \varepsilon_{\gamma} X$, where $X$ is any quantity that does not have any of the relevant indices.

It follows that

$$
P_{\alpha \beta}^{a}=\varepsilon_{\alpha} \varepsilon_{\beta} X^{a}
$$

where $X^{a}$ is an arbitrary quantity except that the indices $\alpha$ and $\beta$ have been extracted. Now using the definition of $P_{\alpha \beta}^{a}$ we see that this means that the entire dependence of $P$ on the fields $\mathrm{A}_{\alpha \beta}^{\prime}$ must occur by virtue of $P$ being a function of 
the combination $F^{a}$ as defined above. However the dependence of $P$ on $\varepsilon$ is not restricted, so that extra $\varepsilon^{\prime} s$ can occur as stated above in Eq. (180). QED.

Acknowledgements. This work and some related work was commenced at Oxford and carried on over a period of years at the CNRS, Marseille; Imperial College, London, Harvard University, Cambridge, MA, and the University of Victoria. I am grateful to C. Becchi, W. Deans, R. Delbourgo, M. Dixon, M. Duff, P. Hawkes, A. Jaffe, T. Kibble, R. Newberry, A. Salam, R. Stora, and J.C. Taylor for valuable remarks and support during this work. I also thank the members of the Department of Physics and Astronomy at the University of Victoria for their generous hospitality.

\section{References}

1. Baulieu, L.: An Introduction to supergravitational anomalies. In: Bardeen, W.A., White, A.R. (eds.). Symposium on anomalies, geometry, topology. (Argonne 1985). Singapore: World Scientific 1985

2. Bonora, L., Cotta-Ramusino, P.: Some remarks on BRS transformations, anomalies and the cohomology of the Lie algebra of the group of gauge transformations. Commun. Math. Phys. 87, 589 (1983)

3. Bonora, L., Cotta-Ramusino, P., Rinaldi, M., Stasheff, J.: The evaluation map in field theory, sigma models and strings. I. Commun. Math. Phys. 112, 237 (1987)

4. Bonora, L., Cotta-Ramusino, P., Rinaldi, M., Stasheff, J.: The evaluation map in field theory, sigma models and strings. II. Commun. Math. Phys. 114, 381 (1988)

5. Breitenlohner, P., Maison, D., Sibold, K.: Renomalization of quantum field theories with non-linear field transformations. Lecture Notes in Physics, Vol. 303. Berlin, Heidelberg, New York: Springer 1988

6. Becchi, C., Rouet, A., Stora, R.: Renormalizable models with broken symmetries. In: Velo, G., Wightman, A.S. (eds.). Renormalization theory (Erice Lectures 1975). Dordrecht: Reidel 1976

7. Becchi, C., Rouet, A., Stora, R.: Renormalization of gauge theories. Ann. Phys. 98, 287 (1976)

8. Becchi, C., Rouet, A., Stora, R.: Renormalization of the abelian Higgs-Kibble model. Commun. Math. Phys. 42, 127 (1975)

9. Brandt, F., Dragon, N., Kreuzer, M.: All consistent Yang-Mills anomalies. Phys. Lett. B231, 263 (1989)

10. Brandt, F., Dragon, N., Kreuzer, M.: All solutions of the consistency equations. To be published in Nucl. Phys. B

11. Ibid: Competeness and nontrivitality of the solutions of the consistency conditions. To be published in Nucl. Phys. B

12. Ibid: Lie algebra cohomology. To be published in Nucl. Phys. B

13. Ibid: The gravitational anomalies. To be published in Nucl. Phys. B

14. Dixon, J.A.: Cohomology and renormalization of gauge theories. I, II, and III. Unpublished preprints 1976-1979

15. Ibid: Calculating BRS cohomology using spectral sequences. In: Solomon, A. (ed.): XVII International Conference on Differential Geometric Methods in Theoretical Physics (Chester, July 1988). Singapore: World Scientific 1989

16. Ibid: How to close the algebra of ten dimensional supersymmetric Yang-Mills theory without using auxiliary fields. UVic preprint 1989

17. Ibid: BRS Cohomology of the supersymmetric chiral multiplet. Commun. Math. Phys.

18. Ibid: Class. Quant. Grav. 7, 1511 (1990)

19. Ibid: Field redefinitions and renormalization of gauge theories. Nucl. Phys. B99, 420 (1975)

20. Deans, W.S., Dixon, J.A.: General theory of renormalization of gauge invariant operators. Phys. Rev. D18A, 1113 (1978)

21. Dubois-Violette, M., Talon, M., Viallet, C.M.: BRS algebras, analysis of the consistency equations of gauge theory. Commun. Math. Phys. 102, 105-122 (1985) 
22. Fadeev, L.D.: Operator anomaly for the Gauss law. Phys. Lett. 145B, 81 (1984)

23. Greub, W., Halperin, S., Vanstone, R.: Connections, curvature and cohomology (3 volumes). New York: Academic Press 1972

24. Green, M., Schwarz, J., Witten, E.: Superstring theory (2 vols). Cambridge, London: Cambridge University Press 1978

25. Joglekar, S.D.: Local operator products in gauge theories. I. Ann. Phys. 108, 233 (1977)

26. Joglekar, S.D.: Local operator products in gauge theories. II. Ann. Phys. 109, 210 (1977)

27. Joglekar, S.D., Lee, B.W.: General theory of renormalization of gauge invariant operators. Ann. Phys. 97, 160 (1976)

28. Kaku, M.: Introduction to superstrings. Berlin, Heidelberg, New York: Springer 1989

29. Kastler, D., Stora, R.: A differential geometric setting for BRS transformations and anomalies. I: J. Geom. Phys. 3, 437-482 (1986); II: J. Geom. Phys. 3, 483-505 (1986)

30. Kastler, D., Stora, R.: Lie-Cartan pairs. J. Geom. Phys. 2, 1 (1985)

31. Kluberg-Stern, H., Zuber, J.B.: Ward identities and some clues to the renormalization of gauge-invariant operators. Phys. Rev. D12, 467 (1975)

32. Kluberg-Stern, H., Zuber, J.B.: Renormalization of non-abelian gauge theories in a background field gauge. I. Phys. Rev. D12, 482 (1975)

33. Kluberg-Stern, H., Zuber, J.B.: Renormalization of non-abelian gauge theories in a background field gauge. II. Phys. Rev. D12, 3159 (1975)

34. Manes, J., Stora, R., Zumino, B.: Algebraic study of chiral anomalies. Commun. Math. Phys. 102, 157 (1985)

35. Ramon Medrano, M., Dixon, J.A.: Anomalies of higher-dimension composite fields. Phys. Rev. D22, 429 (1980)

36. Stora, R.: Continuum gauge theories. In: Levy, M., Mitter, P. (eds.), New developments in quantum field theory and statistical mechanics (Lectures at Cargese 1976). New York, London: Plenum Press 1977

37. Stora, R.: Algebraic Structure and topological origin of anomalies. In: Progress in gauge fields theory. 't Hooft, G., Jaffe, A., Lehmann, H., Mitter, P.K., Singer, I.M., Stora, R. (eds.). New York: Plenum Press 1984

38. Stora, R.: Algebraic structure of chiral anomalies. In: Abat, J., Asorey, M., Cruz, A. (eds.). New perspectives in quantum field theory. Singapore: World Scientific 1986

39. Stora, R.: Differential algebras in field theory: Talk given at the second summer meeting on quantum mechanics of Fundamental Systems. Santiago Chile 1987

40. Talon, M.: BRS algebra and anomalies. In: Lee, H.C., Elias, V., Kunstatter, G., Mann, R.B., Viswanathan, K.S. (eds.). Super field theories. New York, London: Plenum Press 1987

41. West, P.: Introduction to supersymmetry and supergravity, Chap. 7. Singapore: World Scientific 1986

42. Witten, E.: Global anomalies in string theory. In: Bardeen, W.A., White, A.R. (eds.). Symposium on anomalies, geometry, topology (Argonne 1985). Singapore: World Scientific 1985

43. Zinn-Justin, J.: Renormalization of gauge theories. In: Trends in elementary particle theory, Vol. 37. Lecture Notes in Physics. Berlin, Heidelberg, New York: Springer 1975 